\title{
Mpl is activated by dimers of MPN-linked calreticulin mutants stabilized by disulfide bonds and ionic interactions
}

Arunkumar Venkatesan ${ }^{1}$, Jie Geng ${ }^{1}$, Malathi Kandarpa ${ }^{2}$, Sanjeeva Joseph Wijeyesakere ${ }^{1}$, Ashwini Bhide ${ }^{1}$, Moshe Talpaz ${ }^{2}$, Irina D. Pogozheva ${ }^{3}$, and Malini Raghavan ${ }^{1 *}$

${ }^{1}$ Department of Microbiology and Immunology, University of Michigan Medical School, Ann Arbor, MI 48109, USA

${ }^{2}$ Department of Internal Medicine/Division of Hematology/Oncology, University of Michigan Rogel Cancer Center, Ann Arbor, MI 48109, USA

${ }^{3}$ Department of Medicinal Chemistry, College of Pharmacy, University of Michigan, Ann Arbor, MI 48109-1065.

*Corresponding author: Malini Raghavan

E-mail: malinir@umich.edu

Keywords: Calreticulin, Thrombopoietin Receptor (Mpl), Myeloproliferative Neoplasms (MPN), Essential Thrombocythemia, Primary Myelofibrosis, Chaperone, Dimerization. 


\begin{abstract}
Myeloproliferative neoplasms (MPNs) are frequently driven by insertions and deletions within the gene encoding calreticulin $(\mathrm{CRT}) . \mathrm{CRT}_{\mathrm{Del} 52}$ and $\mathrm{CRT}_{\text {Ins5 }}$ are recurrent mutations. Although oncogenic transformation requires both mutated CRT and the myeloproliferative leukemia protein $(\mathrm{Mpl})$, the molecular mechanism of CRT-mediated constitutive activation of $\mathrm{Mpl}$ is unknown. Our studies reveal that the novel $\mathrm{C}$-domain of $\mathrm{CRT}_{\mathrm{Del} 52}$ encodes specificity both for Mpl binding and for disulfide-mediated CRT dimerization. Disulfidestabilized CRT $\mathrm{Del}_{\text {De }}$ dimers and multimers are observed in MPN patient-derived platelet lysates and in transfected mammalian cells. Cysteine mutations within both the novel Cdomain (C400A and C404A) and the conserved N-domain (C163A) of $\mathrm{CRT}_{\mathrm{Del52}}$ are required to reduce disulfide-mediated dimers and multimers of $\mathrm{CRT}_{\mathrm{Del} 52}$. Based on these data and published structures of crystalized CRT oligomers, we tested the relevance of ionic interactions between charged residues proximal to $\mathrm{C} 163$ at the $\mathrm{N}$-domain dimerization interface. Charge alteration at these residues affected dimerization and multimerization of both wild type and $\mathrm{CRT}_{\text {Del52. Elimination of intermolecular disulfides }}$ and disruption of ionic interactions at both proposed dimerization interfaces was required to abrogate the ability of $\mathrm{CRT}_{\mathrm{Del} 52}$ to induce cytokine-independent cell proliferation via Mpl. Based on these findings, we propose a structural model of the Mpl-activating $\mathrm{CRT}_{\text {Del52 }}$ unit as a covalently-linked dimer that is stabilized by disulfides and ionic interactions at both the C-domain and N-domain. MPNs exploit a natural dimerization interface of CRT combined with C-domain gain-of-functions to achieve cell transformation.
\end{abstract}




\section{Introduction}

Myeloproliferative neoplasms (MPNs) comprising Polycythemia Vera (PV), Essential Thrombocythemia (ET) and Primary Myelofibrosis (MF) are hematopoietic stem cell disorders characterized by the overproduction of myeloid lineage cells (reviewed in reference (1)). Somatic mutations resulting from deletions or insertions in exon 9 of the CALR gene were identified in the majority of patients with PV, ET, and MF who were negative for mutations in Janus Kinase 2 (JAK2) and in the thrombopoietin receptor/myeloproliferative leukemia protein $(\mathrm{Mpl})(2,3)$. A majority of the CALRmutated patients have one of two gene variants: type 1 with a $52 \mathrm{bp}$ deletion (Del52) or type 2 with a 5 bp insertion (Ins5) $(2,3)$. The mutations change the sequence of the acidic C-terminus of CRT to a basic sequence, and cause loss of the endoplasmic reticulum (ER) retention KDEL sequence $(2,3)$.

Calreticulin (CRT) is an ER calcium-binding chaperone that functions in the folding and assembly of glycoproteins $(4,5)$. CRT contains three domains, a lectin-like N-terminal domain (N-domain) containing the glycan and high-affinity calcium binding sites $(6,7)$, an elongated hairpin-like $\mathrm{P}$ domain containing the co-chaperone-binding site (8) and a mainly $\alpha$-helical acidic C-terminal domain (C-domain), which contains multiple lowaffinity/ $\mathrm{Ca}^{2+}$ binding sites (9-11). CRT has specificity for monoglucosylated N-glycans on substrate proteins, which are transiently acquired during glycoprotein maturation in the ER.

MPN-linked CRT mutants induce specific amplification of the megakaryocyte lineage of cells and increase platelet production (12-16). CRT mutants mediate constitutive activation of $\mathrm{Mpl}$ and downstream signaling pathways (12-15, 17-20). Studies of the direct interaction between $\mathrm{Mpl}$ and MPN-linked CRT mutants by co-immunoprecipitation analyses demonstrated a critical role of glycan-binding site residues of CRT $(13,20,21)$ in the recognition of sugars linked to $\mathrm{N} 117$ of $\mathrm{Mpl}(13,22)$. Since both wild type and mutant CRT are, in principle, capable of glycan-mediated interactions with Mpl, glycan-binding alone cannot account for mutant CRT-mediated Mpl activation. The novel basic amino acids in the C-termini of mutant CRTs were shown to be also critical for inducing cell proliferation and developing the ET phenotype $(15,18,19)$. Studies of truncated mutants 
revealed that residues 376-383 from the C-terminus of $\mathrm{CRT}_{\mathrm{Del} 52}$ are required to activate Mpl-mediated signaling (20). However, the molecular contacts between Mpl and the Cterminal tail of CRT mutants remain undefined. Here we demonstrate a direct role of the C-terminal tail of $\mathrm{CRT}_{\text {Del52 }}$ in $\mathrm{Mpl}$ binding and in conferring Mpl specificity to $\mathrm{CRT}_{\text {Del52. }}$.

Ligand-induced dimerization of receptor molecules is an established paradigm for signal transduction mediated by cytokine receptors $(23,24)$ including the erythropoietin (Epo) receptor $(25,26)$, and $\mathrm{Mpl}(27)$. While multimeric forms of MPN mutant CRT have been described and implicated in Mpl activation and cytokine-independent cell growth (18, 28), the nature of the productive multimeric forms of CRT mutants that may trigger Mpl dimerization and activation has not been established. Here, based on the occurrence of novel cysteine residues in the CRT mutant C-termini $(2,3)$, we investigated the relevance of disulfide bond-mediated interactions in CRT multimerization in primary patient platelets and human cell lines expressing recombinant mutant CRT. In addition, we tested the relevance of non-covalent interactions relevant to CRT multimerization.

\section{Results}

\section{Mutant CRTs form disulfide-stabilized multimers in MPN patient platelets and are detectable in MPN patient serum}

To assess mutant CRT multimerization in primary cells, which has not previously been undertaken, platelets were purified from the blood of healthy donors or MPN patients with the known clinical characteristics summarized in Table S1 and analyzed by immunoblots. An antibody (anti-CRT $\left(\mathrm{C}_{\text {mut }}\right)$ ) was raised against the C-terminal 22 residues of mutant CRT, within the novel mutant-specific C-termini, to examine MPN mutant CRT expression and oligomerization. The specificity of the antibody was established using purified proteins (Figure $\mathrm{S} 1$ ). Anti-CRT $\left(\mathrm{C}_{\mathrm{mut}}\right)$ specifically detected proteins in platelet lysates from patients with CRT mutants but not from patients with JAK2 mutants or healthy control individuals (Figure 1A and Figure S2A, top panel). As expected, based on size, mutant CRT in samples 
8744 and 4995-2 which correspond to $\mathrm{CRT}_{\text {Del4 }}$ and $\mathrm{CRT}_{\text {Ins } 5}$ mutations, migrate more slowly than the CRT $\mathrm{Del}_{\mathrm{D} 2}$ samples in the same blots (Figure 1A and S2A, top panel). The levels of mutant CRT expression were somewhat variable between patients. For example, platelet lysates from consecutive blood collections from the patient 2648 consistently indicated high expression of the mutant CRT relative to other patients (Figure S2A for 2648-2).

In contrast to anti-CRT $\left(\mathrm{C}_{\text {mut }}\right)$, anti-CRT(N), a commercial antibody from Cell Signaling Technology (CST) directed against the N-terminus of CRT, detected CRT in all samples (Figure 1B and Figure S2A, middle panels). As discussed below, in transfected

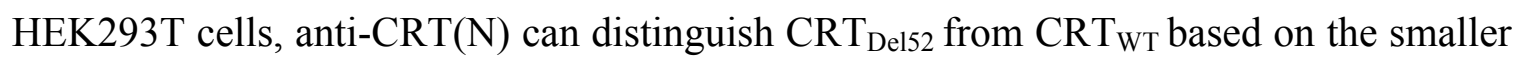
size of $\mathrm{CRT}_{\text {Del52. }}$. However, two distinct $\mathrm{CRT}$ bands were not readily detectable with antiCRT(N) in lysates from MPN patient platelets (Figure 1B and Figure S2A and S2B, middle panels, except possibly for the most highly expressed samples such as 2648-2), indicating that the detected protein corresponds to $\mathrm{CRT}_{\mathrm{WT}}$. The C-terminal frameshift in all MPN mutant CRT results in a loss of the ER retention KDEL motif, causing their secretion from cells (29-32) and indeed the mutants but not wild type CRT are detectable in patient serum by co-immunoprecipitation analyses (Figure 1D). Enhanced secretion is expected to render all MPN CRT mutants more difficult to detect than the wild type CRT using generic antiCRT antibodies in platelet lysates.

Analyses of the platelet lysates from different patients on non-reducing gels indicated that the mutant CRT species are DTT-sensitive which was also most readily apparent in immunoblots with anti-CRT $\left(\mathrm{C}_{\mathrm{mut}}\right)$ for most samples, and with both anti$\mathrm{CRT}\left(\mathrm{C}_{\text {mut }}\right)$ and anti-CRT(N) for the high-expressing samples 2648-3 and 8251-2 (Figures 1A-B and Figure S2B; reducing gels (+DTT) compared with non-reducing gels (-DTT)). Notably, a band consistent with a dimer of CRT ( 100 KDa) was visualized in the nonreducing gels with anti-CRT $\left(\mathrm{C}_{\text {mut }}\right)$ in several mutant $\mathrm{CRT}$ samples, in addition to several additional high molecular weight species (Figure 1A and S2B, top panel).

We also conducted immunoblots of the platelet lysates following native-PAGE. High molecular weight complexes, migrating close to the stacking gel, were clearly detectable with both anti-CRT $\left(\mathrm{C}_{\text {mut }}\right)$ and anti-CRT(N) in the CRT mutant samples (Figure 1E-F and Figure S2C-D). Some platelet preparations show a band above the $64 \mathrm{kDa}$ marker that likely represents a contamination of hemoglobin $(\mathrm{Hb})$, based on the appearance of the 
same background in red blood cell (RBC) preparations (Figure 1E-F boxed; Figure S2D shows the RBC lysate), possible arising from $\mathrm{RBC}$ contamination in the platelet preparations. Thus, CRT mutants form disulfide-bonded high molecular weight complexes in patient platelets.

\section{The C-domain of $\mathrm{CRT}_{\text {Del52 }}$ confers specificity for $\mathrm{Mpl}$ and forms disulfide-linked dimers}

The novel C-domains of type-I and type-II MPN mutant CRT contain two or three cysteine residues (Figure 2A). To further study the C-domain multimerization and function, we generated C-domain fusions with the B1 domain of protein $\mathrm{G}$ (GB1; which is used to increase the yield and solubility of small proteins $(33,34))$ and $6 x$ histidine (his) tags. The C-domains of $\mathrm{CRT}_{\mathrm{WT}}, \mathrm{CRT}_{\mathrm{Del52}}$, and different truncation mutants of $\mathrm{CRT}_{\text {Del52 }}$ (Figure $2 \mathrm{~A}$ ) were expressed as His-GB1-tagged proteins in HEK293 T cells (Figure 2B). All constructs encoded the signal sequences of Cox 2 to allow insertion into the ER lumen. Since the $\mathrm{CRT}_{\text {De152 }}$ mutation was the most frequent in our patient group (Table S1), our studies were largely focused on this mutant. Using the anti-His antibody, we found that the C-domain of $\mathrm{CRT}_{\mathrm{Del} 52}$ was expressed at higher levels than the C-domain of $\mathrm{CRT}_{\mathrm{WT}}$ (Figure 2B, lane 1 compared to lane 2). Furthermore, truncations of $\mathrm{CRT}_{\text {Del52 }} \mathrm{C}$-domain progressively reduced expression levels, with expression essentially undetectable for the C-domain of $\mathrm{CRT}_{\text {Del52 } 336}($ Figure 2B, lanes 2-6).

We performed co-IP assays in HEK293T cells co-transfected with plasmids encoding $\mathrm{Mpl}$ and the various $\mathrm{C}$-domain constructs. Binding interactions were observed between $\mathrm{Mpl}$ and the isolated $\mathrm{C}$-domains of all the $\mathrm{CRT}_{\mathrm{Del52}}$ constructs, except the poorly expressed $\mathrm{CRT}_{\text {Del52 } \triangle 36}$, whereas binding between $\mathrm{Mpl}$ and the $\mathrm{C}$-domain of $\mathrm{CRT}_{\mathrm{WT}}$ was not observed (Figure 2B, lanes 7-12). The absence of C-domain bands in the lysate+beads controls suggests that binding of $\mathrm{C}$-domains to $\mathrm{Mpl}$ is specific, as protein $\mathrm{G}$ contained on the beads is not bound by the C-domain constructs (Figure 2B, lanes 16-19).

The findings of Figure 2B parallel the previous findings of preferential interactions between the full-length versions of MPN mutant CRT and Mpl in the steady state $(12,13$, 18-22). Correspondingly, in an anti-Mpl co-IP with HEK293T cells transfected with plasmids encoding Mpl and full-length mutant CRT constructs, binding interactions were 
readily detectable between $\mathrm{CRT}$ mutants and $\mathrm{Mpl}$ using anti-CRT $\left(\mathrm{C}_{\mathrm{mut}}\right)$ (Figure $\mathrm{S} 3 \mathrm{~A}$, top and middle panels for $\mathrm{Mpl}$ and anti-CRT $\left(\mathrm{C}_{\mathrm{mut}}\right)$ blots). On the other hand, $\mathrm{CRT}_{\mathrm{WT}}$ signals (probed with anti-CRT(Thermo), a commercial polyclonal antibody directed against the whole protein as immunogen; Thermo Fisher), were essentially undetectable in the steady state, following anti-Mpl co-IPs (Figure S3A, lower panel). Wild type but not mutant protein over-expression is detectable with anti-CRT(Thermo) (Figure S3A, compare lanes 1-3 with lane 4). Again, as noted above, anti-CRT $\left(\mathrm{C}_{\text {mut }}\right)$ allows for more sensitive detection of the mutants, and additionally, it is possible that anti-CRT(Thermo) epitopes reside within the C-terminus. Preferential binding between mutant CRT and Mpl was also detectable in patient platelets (Figures S3B and S3C).

In lysates of transfected HEK293T cells, a band consistent with the size of a Cdomain dimer was detected under non-reducing conditions, for the $\mathrm{CRT}_{\text {Del52-C }}$ but not its cysteine mutant, $\mathrm{CRT}_{\text {Del52-C(C44A/C48A) }}$ (Figure 2C, right panel, lanes 2 and 3). Thus, the mutant C-domain, when expressed on its own, is capable of forming disulfide-linked dimers.

For further functional assessments of the C-domain constructs, we first used retroviral infections to stably express $\mathrm{Mpl}$ in $\mathrm{Ba} / \mathrm{F} 3$ cells, and further expressed $\mathrm{CRT}_{\mathrm{Del}} 2$ or its $\mathrm{C}$-domain or cysteine mutated $\mathrm{C}$-domain. Binding interactions between $\mathrm{Mpl}$ and the $\mathrm{CRT}_{\text {Del52 }}$ full-length and C-domain constructs were observed in $\mathrm{Ba} / \mathrm{F} 3 \mathrm{Mpl}$ cells (Figure S4A), as was observed in HEK293T cells (Figure 2B). The efficiency of Mpl binding to the C-domain constructs appears to be at least as high as that for full-length $\mathrm{CRT}_{\text {Del52 }}$ (Figure S4A; lane 2 compared with lane 4), even though expression of the C-domain constructs is not directly detectable in $\mathrm{Ba} / \mathrm{F} 3$ lysates. MPN mutant CRT constructs are known to induce proliferation of $\mathrm{Ba} / \mathrm{F} 3$ cells in a Mpl-dependent and cytokine-independent manner $(13,22)$, as also shown in Figure S4B. Consistent with the previous findings (19), the $\mathrm{CRT}_{\mathrm{Del52}} \mathrm{C}$-domain alone is insufficient to mediate proliferation (Figure S4B). Thus, while the $\mathrm{CRT}_{\mathrm{Del52}} \mathrm{C}$-domains confer $\mathrm{Mpl}$ binding specificity and dimerize (Figure 2), additional interactions mediated by $\mathrm{CRT}_{\mathrm{Del} 52}$ are required for functional interactions with Mpl (Figures S4). Notably, the $\mathrm{CRT}_{\text {Del52 }} \mathrm{C}$-domain has higher stability than the wild type C-domain in HEK293T cells, based on relative expression levels in lysates, both in the 
presence and in the absence of Mpl (Figure 2B and S4C respectively), consistent with the higher predicted helical content of the $\mathrm{CRT}_{\mathrm{Del} 52} \mathrm{C}$-domain (Figure $2 \mathrm{~A}$ ).

\section{Truncations of C-terminal cysteines of full-length $\mathrm{CRT}_{\text {Del52 }}$ alter but do not abrogate disulfide-linked interactions}

To ask if C-domain cysteines were sufficient for full-length $\mathrm{CRT}_{\text {Del52 }}$ multimerization, we generated the successive truncations of the C-terminal sequences (Figure 2A) within fulllength $\mathrm{CRT}_{\mathrm{Del} 52}$ and expressed those constructs as N-terminal His and GB1-tagged (hisGB1) proteins. $\mathrm{CRT}_{\text {Del52 } 12}$ and $\mathrm{CRT}_{\text {Del52 } 19}$ were expressed at low levels and their protein loads had to be increased to achieve similar protein expression as the other constructs (Figure 3A; note varying intensities of the endogenous CRT bands). Furthermore, in nonreducing SDS gels (Figure 3B), only $\mathrm{CRT}_{\mathrm{WT}}$ and $\mathrm{CRT}_{\text {Del52 } 236}$ were largely monomeric. Bands consistent with the size of dimers/multimers were observed for $\mathrm{CRT}_{\mathrm{Del} 52}$,

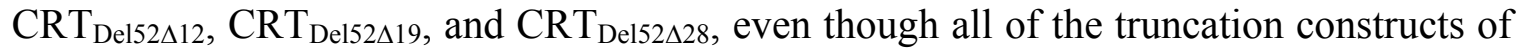
$\mathrm{CRT}_{\text {Del52 }}$ lacked the two C-terminal cysteines. However, the specific band indicated as

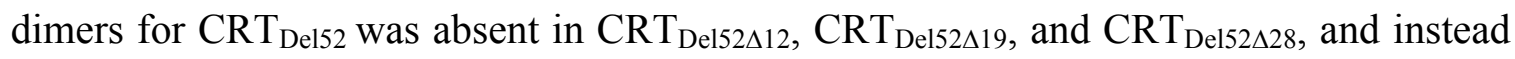
slower mobility bands were observed (Figure 3B, lane 3, compared to lanes 4-6). These findings suggested that the presence of two $\mathrm{C}$-terminal cysteines in $\mathrm{CRT}_{\text {Del52 }}$ contribute to the induction of distinct disulfide-linked species. Additionally, the proportion of monomer bands to total CRT bands progressively increases with increased truncation size (quantification of 3B is shown in Figure 3C). In native gels, only multimers were detectable for $\mathrm{CRT}_{\mathrm{Del52 \Delta 12}}$ and $\mathrm{CRT}_{\mathrm{Del} 52 \Delta 19}$, monomers and multimers were detected for $\mathrm{CRT}_{\mathrm{Del} 52 \Delta 28}$, whereas $\mathrm{CRT}_{\text {Del52 } 236}$ migrated largely as monomers (Figure 3D). Together the findings of Figure 3 suggested that novel $\mathrm{C}$-domain cysteines and additional cysteines contribute to the formation of $\mathrm{CRT}_{\mathrm{Del} 52}$ disulfide-linked dimers. Further, the $\mathrm{CRT}_{\mathrm{Del} 52 \Delta 28}$ truncation is needed to partially destabilize $\mathrm{CRT}_{\text {Del52 }}$ multimers and the $\mathrm{CRT}_{\text {De152 } 36}$ truncation is needed to fully destabilize $\mathrm{CRT}_{\text {Del52 }}$ multimers, suggesting that both covalent and non-covalent interactions mediated by the C-domains contribute to multimer formation. 


\section{Disulfide-linked CRT Del52 $_{2}$ dimer and multimer formation is C-domain and N-domain dependent}

To further elucidate the mode of $\mathrm{CRT}_{\text {Del52 }}$ multimerization, we generated various cysteine mutants of $\mathrm{CRT}_{\mathrm{Del52}}$ as untagged constructs and examined their multimerization in transfected HEK293T cells. Anti-CRT $\left(\mathrm{C}_{\mathrm{mut}}\right)$ did not detect wild type CRT expressed in HEK293T cells and was specific for the mutants (Figure 4A). As noted above, CRT $_{\text {Del52 }}$ formed dimers and higher order species, which were detected in immunoblots under nonreducing conditions (Figure 4B, lanes 3-4). $\mathrm{CRT}_{\text {Del52-2CA }}\left(\mathrm{CRT}_{\text {Del52(C400A/C404A), that lacks }}\right.$ the novel cysteines in the mutant C-terminus) formed fewer higher order multimer structures and more lower order structures compared with $\mathrm{CRT}_{\text {Del52 }}$ (compare Figure 4B lanes 5 and 6, which had similar protein loads as shown in Figure 4A, lanes 5 and 6, and quantification in 4C). Notably, the bands indicated as dimers (which migrated at approximately $100 \mathrm{kDa}$, the expected size for Del52 dimers) were more intense but migrated more slowly for $\mathrm{CRT}_{\mathrm{Del} 52-2 \mathrm{CA}}$ compared to the corresponding $\mathrm{CRT}_{\text {De152 }}$ construct (Figure 4B, lanes 3-4 compared with 5-6), suggesting that the $\mathrm{CRT}_{\mathrm{Del} 52}$ dimers are rendered more compact by the presence of the two C-terminal cysteines, C400 and C404. CRT has a single disulfide bond between $\mathrm{C} 105$ and $\mathrm{C} 137$ within its globular domain. C163, the only free cysteine in the wild type CRT that is also present in MPN-linked CRT mutants. Alasubstitution of this residue in the $\mathrm{CRT}_{\text {De152-CA }}\left(\mathrm{CRT}_{\text {Del52(C163A) }}\right)$ mutant resulted in a dimer and multimer pattern similar to $\mathrm{CRT}_{\mathrm{Del} 52}$. Notably, the intensities of bands indicated as monomers and dimers were stronger for $\mathrm{CRT}_{\text {Del52-CA }}$ compared with $\mathrm{CRT}_{\text {Del52, whereas }}$ bands indicated as multimers were stronger for $\mathrm{CRT}_{\text {Del52 }}$ compared with $\mathrm{CRT}_{\text {Del52-CA }}$ (Figure 4B, lanes 9-10 compared with lanes 3-4 and quantification in Figure 4C), under conditions of similar protein loads (Figure 4A, lanes 9-10 compared with lanes 3-4). Additionally, monomer species were predominant for the triple cysteine mutant $\mathrm{CRT}_{\text {Del52- }}$ 3CA $\left(\mathrm{CRT}_{\operatorname{Del} 52(\mathrm{C} 163 \mathrm{~A} / \mathrm{C400A} / \mathrm{C} 404 \mathrm{~A})}\right)$ indicating that all three mutations were needed to significantly inhibit the formation of disulfide-linked dimers and multimers (Figure 4B, lanes 7-8 and Figure 4C). C105 and C137 are the only remaining cysteines in CRT Del52-3CA, $_{\text {, }}$ residues that form a disulfide bond in CRT structures $(6,7)$. The disulfide linked dimers and multimers still observable with $\mathrm{CRT}_{\text {De152-3CA }}$ likely correspond to low efficiency thioldisulfide exchange between the monomer subunits of dimers/multimer. 
Parallel immunoblots under reducing conditions with anti-CRT(N) indicated that all $\mathrm{CRT}_{\text {Del52 }}$ constructs migrate more rapidly than $\mathrm{CRT}_{\mathrm{WT}}$, consistent with their smaller size (Figure 4D). Additionally, bands indicated as dimeric and high order oligomeric CRT structures were detectable under non-reducing conditions similarly to those detected with anti-CRT $\left(\mathrm{C}_{\text {mut }}\right)$ (Figure $4 \mathrm{E}$ ). Slower migration of $\mathrm{CRT}_{\text {Del52-2CA }}$ dimer bands than $\mathrm{CRT}_{\text {Del52 }}$ (Figure 4E, lanes 3-4 compared with 5-6), and higher representation of dimer bands relative to higher order multimers for $\mathrm{CRT}_{\text {Del52-CA }}$ compared with $\mathrm{CRT}_{\text {Del52 }}$ were again rather noticeable (Figure 4E, lanes 3-4 compared with 9-10). Finally, disulfide-linked multimeric structures were poorly detected for the triple mutant $\mathrm{CRT}_{\text {Del52-3CA. Together, these finding }}$ implicate C-terminal cysteines (C400 and $\mathrm{C} 404)$ and $\mathrm{C} 163$ in disulfide-mediated dimerization and multimerization of $\mathrm{CRT}_{\text {De152 }}$ mutant.

In native-PAGE gels, however, all four $\mathrm{CRT}_{\mathrm{Del52}}$ constructs formed higher order species (Figures 4F). Nonetheless, signals corresponding to monomeric species were readily detectable only in the $\mathrm{CRT}_{\text {Del52-2CA }}$ and $\mathrm{CRT}_{\text {Del52-3CA }}$ lysates (Figure $4 \mathrm{~F}$ ). We concluded from these analyses that both covalent and non-covalent interactions contribute to $\mathrm{CRT}_{\text {Del52 }}$ multimerization. Together with the truncation mutant data (Figure 3), these findings indicate that disulfide-dependent interactions contribute to dimer and multimer stability, but that loss of S-S bonds is not sufficient to fully block multimer formation.

Reducing and non-reducing SDS-PAGE analyses indicated the formation of disulfidelinked oligomers for $\mathrm{CRT}_{\text {Ins } 5}$ constructs, similarly to that observed for $\mathrm{CRT}_{\text {Del52, although }}$ the overall pattern was more complex than that of $\mathrm{CRT}_{\text {Del52 }}$ because of the presence of an additional C-terminal cysteine in $\mathrm{CRT}_{\text {Ins5 }}$ mutant (Figure 2A and Figure S5). Further mutational studies were focused on $\mathrm{CRT}_{\text {Del52 }}$ mutant.

\section{A working model for a $\mathrm{CRT}_{\text {Del52 }}$ dimer including ionic interactions mediated by the}

\section{N-domain}

We generated a model for monomeric $\mathrm{CRT}_{\text {Del52 }}$ based on the high resolution crystal structure of the human CRT (35) for the N-domain and the proximal part of C-domain (residues 19-203 and 303-366, excluding the P-domain). The C-domain $\alpha$-helix was further extended to K386 using the structure of the human major histocompatibility complex class 
I peptide-loading complex which includes CRT (11). The distal C-terminal segment (residues 399-406) of $\mathrm{CRT}_{\text {Del52 }}$ containing C400 and C404 was modeled as a 2-turn $\alpha$-helix based on secondary structure predictions by I-TASSER (Figure 2A) while the connecting loop (residues 387-398) was modeled in an extended conformation.

We next examined various crystal structures of CRT for dimerization modes that could account for the experimental data (Figures 1-4). Among the structures (see supplemental methods for details), the crystal structure of a 10-mer of human CRT (D71K mutant; PDB ID: 5lk5) (35) revealed stable dimers formed through tight packing of antiparallel $\alpha$-helices from C-domains (Figure S6A, "C-C" dimer) or through intermolecular ionic interactions between N-domain loop residues 160-167 (Figure S6B, "N-N" dimer). This N-domain loop contains C163, which, based on our data (Figure 4), is involved in disulfide-mediated dimerization. In the crystal structure of the "N-N" dimer, two C163 are not in direct contact, but could move closer to each other and form a disulfide bond following minor loop rearrangements. In addition, the C-termini of molecules in the " $\mathrm{N}-\mathrm{N}$ "-dimer are much closer to each other than in the "C-C"-dimer, and, therefore, the " $\mathrm{N}-\mathrm{N}$ "-dimer can be additionally connected through two intermolecular disulfides between C400 and C404 of $\mathrm{CRT}_{\text {Del52 }}$ (Figures 2-4). Two negatively charged D165 residues from both subunits of the "N-N" dimer form hydrogen bonds and four intermolecular ionic pairs with positively charged K142 and R162 from the opposite subunits. Closer examination of the "N-N" dimerization mode of $\mathrm{CRT}_{\text {Del52 }}$ mutants (Figure 5A) allows prediction of two additional symmetrical "N-C" dimerization interfaces that could be formed between $\mathrm{N}$-domain of one molecule and C-domain of another molecule. A slight decrease of the $\alpha$-helix kink at A352 would move the C-terminal part of the $\alpha$-helix (residues 366-383) closer to N-domain glycan recognition site. As a result, dimer-stabilizing ionic and hydrophobic interactions may be formed between positively charged and non-polar residues from C-domain of one molecule and negatively charged and aromatic residues from the $\mathrm{N}$-domain glycan-binding site of the second molecule. The existence of ionic or hydrophobic interactions between these residue pairs may explain the significant role of residues 376-383 for multimerization of $\mathrm{CRT}_{\text {Del52 }}$ constructs, as their truncation in $\mathrm{CRT}_{\text {Del52 } 236}$ eliminated oligomer formation (Figure 3). Thus, the "N-N" dimerization mode is compatible with our experimental data (Figures 2-4). Furthermore, $\mathrm{CRT}_{\text {Del52 }}$ tetramers and larger oligomers can be easily formed 
by combining both types of dimerization modes: through C-domain helix-helix interface and N-domain loop-loop interface (Figure S6C).

We previously showed that the H170A mutant of murine CRT (H153A in mature protein numbering) forms dimers at enhanced levels, visualized by native-PAGE of the purified protein (36). H170 is at predicted dimer interface in the model shown in Figure 5A and forms intramolecular interactions with D166. We found that H170A mutations of wild type human CRT also induce disulfide-linked dimers and multimers, visualized in transfected HEK cells (Figures 5B and 5C), indicating the potential relevance of the dimer model of Figure 5A to multimeric interactions in both wild type CRT and its MPN mutants. We and others have also previously reported that disulfide-linked dimers of CRT are induced by heat-shock, calcium depletion and other destabilizing conditions $(37,38)$.

Based on the dimer model of Figure 5A, in addition to the covalent interactions, $\mathrm{CRT}_{\text {De152 }}$ dimers are stabilized by two sets of D165-K142 and D165-R162 salt bridges at the globular domain dimer interface. The $\mathrm{D} 165 \mathrm{~K}$ mutations alone $\left(\mathrm{CRT}_{\text {Del52-D165K }}\right)$ did not significantly influence the oligomerization potential of CRT $\mathrm{Del}_{52}$ (Figure S7). However, the combination of $\mathrm{D} 165 \mathrm{~K}$ and $\mathrm{CRT}_{\text {Del52-3CA }}$ mutations induced more monomeric species in the $\mathrm{CRT}_{\text {De152-3CA/D165K }}$ mutant compared with $\mathrm{CRT}_{\text {Del52-3CA }}$ in native blots, but not in nonreducing blots (Figures 6A and 6B), indicating the impact of combined disruptions of both dimer interfaces. This was not observed with the D166K mutation, for which enhanced levels of disulfide-linked species were induced (with both $\mathrm{CRT}_{\text {Del52-3CA/D166K }}$ and the double mutant $\mathrm{CRT}_{\text {De152-3CA/D165KD166K }}\left(\mathrm{CRT}_{\text {Del52-3CA-2DK }}\right)$ compared with $\mathrm{CRT}_{\text {Del52-3CA }}$ (Figures 6A and 6B). Since the only remaining cysteines in all $\mathrm{CRT}_{\mathrm{Del} 52-3 \mathrm{CA}}$ constructs are C105 and C137, which form an intramolecular disulfide in wild type CRT $(6,7)$, these findings suggest that repulsion between several interacting positively charged residues in the D166K mutant following disruption of its interaction with H170, induce rearrangements that bring the $\mathrm{C} 105-\mathrm{C} 137$ disulfide from one subunit of the N-N dimer closer (from $18 \AA$ to $5 \AA$ ) to that from the other subunit to enhance intermolecular disulfides via thiol-disulfide exchange. Correspondingly, the H170A mutation on the $\mathrm{CRT}_{\text {Del52-3CA }}$ background also induces disulfide-linked dimers and multimers (Figures 6C and 6D), that are expected to correspond to enhanced intermolecular disulfides mediated by $\mathrm{C} 105-\mathrm{C} 137$ rearrangements. Overall, the findings of Figure 6 support the relevance of the dimer model 
of Figure 5A to Del52 multimerization, and suggest that MPN mutations exploit a natural dimerization interface of CRT known to be induced by ER stress conditions.

\section{Large C-domain truncations or combined $\mathrm{N}$-domain and $\mathrm{C}$-domain dimer interface mutations are required to abrogate $\mathrm{CRT}_{\text {Del52-mediated cell proliferation }}$}

$\mathrm{Ba} / \mathrm{F} 3-\mathrm{Mpl}$ cells were transduced with viruses encoding the series of untagged C-domain truncated constructs of $\mathrm{CRT}_{\mathrm{Del} 52}$ and its point mutants to compare their proliferationinducing activities. We observed reduced abilities of $\mathrm{CRT}_{\mathrm{Del} 52 \Delta 12}$ and $\mathrm{CRT}_{\mathrm{Del} 52 \Delta 19}$ to mediate $\mathrm{Ba} / \mathrm{F} 3$ cell proliferation (although statistically non-significant), while $\mathrm{CRT}_{\mathrm{Del} 52 \Delta 28}$, $\mathrm{CRT}_{\text {Del52 } 236}$ were unable to promote cell growth, similar to $\mathrm{CRT}_{\mathrm{WT}}$ (Figure 7A; all constructs shown in Figure 7 are untagged). These results deviate from those of Elf et al (20), where the transforming capacity of CRT mutant was abolished only after the most

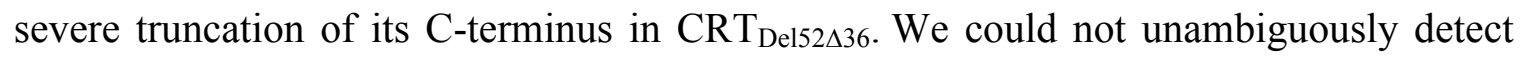
expression of the truncated untagged CRT constructs over interfering background bands in two independent sets of retroviral infection of $\mathrm{Ba} / \mathrm{F} 3-\mathrm{Mpl}$ cells (data not shown). Thus, we re-assessed expression and functional activities of N-terminal histidine and GB1-tagged versions in $\mathrm{Ba} / \mathrm{F} 3-\mathrm{Mpl}$ cells following plasmid nucleofections (Figure S8). Proliferation mediated by tagged versions of $\mathrm{CRT}_{\mathrm{Del} 52 \Delta 28}$ and $\mathrm{CRT}_{\text {Del52 } 236}$ was again impaired, under conditions where expression of both those constructs was detectable at higher levels than of $\mathrm{CRT}_{\text {Del52 }}$ (Figure S8). Thus, while removal of the novel C-terminal cysteines has a small effect on $\mathrm{CRT}_{\text {De152 }}$ mediated proliferation, a larger truncation is needed to completely abrogate $\mathrm{CRT}_{\text {Del52 }}$ mediated proliferation (Figure 7A and $\mathrm{S} 8$ ).

Both $\mathrm{CRT}_{\text {De152-2CA }}$ and $\mathrm{CRT}_{\text {De152-3CA }}$ showed non-significant reductions in the ability to induce cytokine-independent proliferation of $\mathrm{Ba} / \mathrm{F} 3-\mathrm{Mpl}$ cells compared with $\mathrm{CRT}_{\mathrm{Del52}}$, (Figure 7B), despite the higher expression of the mutant (Figure 7E, top panel, lysate lanes). Notably, we also observed decreased binding of $\mathrm{CRT}_{\text {Del52-2CA }}$ and $\mathrm{CRT}_{\text {Del52-3CA }}$ to $\mathrm{Mpl}$ relative to $\mathrm{CRT}_{\text {Del52 }}$ despite the higher expression of the mutants (Figure 7E, lanes marked as IP). There was also a non-significant reduction in $\mathrm{Ba} / \mathrm{F} 3-\mathrm{Mpl}$ cell proliferation induced by $\mathrm{CRT}_{\text {De152-D165K }}$ and $\mathrm{CRT}_{\text {De152-D166K }}$ compared with $\mathrm{CRT}_{\text {Del52 }}$ (Figure $7 \mathrm{C}$ ). Notably, the combination of $\mathrm{D} 165 \mathrm{~K}$ or $\mathrm{D} 166 \mathrm{~K}$ or both with the $\mathrm{CRT}_{\mathrm{Del} 52-3 \mathrm{CA}}$ mutation resulted in marked abolishment of cell proliferation (Figure 7C). The impaired abilities of 
$\mathrm{CRT}_{\text {Del52-3CA/D165K }}$ and $\mathrm{CRT}_{\text {Del52-3CA/D166K }}$ to mediate cytokine-independent cell proliferation correlated with almost complete impairment in Mpl binding (Figure 7F, IP lanes). Parallel results were obtained with $\mathrm{CRT}_{\text {Del52-3CA/H170A }}$ (Figure 7D and 7G).

Among all mutants, the expression level of the combined mutant $\mathrm{CRT}_{\text {Del52-3CA/2DK }}$ $(3 \mathrm{CA}+\mathrm{D} 165 \mathrm{~K}+\mathrm{D} 166 \mathrm{~K})$ was rather low. Thus, the impaired cell proliferation induced by this mutant could be partially caused by its low expression. In contrast, the expression of $\mathrm{CRT}_{\text {Del52-3CA }}, \mathrm{CRT}_{\text {Del52-3CA/D165K }}$ and $\mathrm{CRT}_{\text {Del52-3CA/D166K }}, \mathrm{CRT}_{\text {Del52-3CA/H170A }}$ mutants were higher than of $\mathrm{CRT}_{\mathrm{Del52}}$ (Figures $7 \mathrm{~F}$ and $7 \mathrm{G}$, top panels), indicating an enhanced stability of these mutants. Therefore, loss of stability could not explain their functional loss.

\section{Discussion}

In this work, we observed that the presence of the novel C-terminal domain in MPN mutant CRT induces CRT dimerization and formation of higher order oligomers in pathologically relevant MPN patients-derived platelets (Figure 1), and in transfected HEK293T cells (Figures 2-4). Absence of oligomeric bands under disulfide-reducing conditions indicates that the intermolecular disulfides stabilize multimers both in transfected cells and in MPN patient-derived platelets.

The data presented here are consistent with the model of dimers of CRT $\mathrm{Del}_{52}$ with two dimerization interfaces, the first involving interactions between the distal parts of Cterminal tails and the second involving association of globular domains via loop residues 160-167 (Figure 5A). The novel C-domains of the MPN-linked CRT mutant, CRT $_{\text {Del52, }}$ contains two cysteine residues, C400 and C404, (Figure 2A), whose mutations to alanines abrogate the formation of dimers of isolated C-domains (Figure 2C). In addition, C163 residue from the globular $\mathrm{N}$-domain contributes to the formation of intermolecular disulfides. Indeed, a combination of mutations of two C-terminal cysteines and the C163A mutation in the full-length $\mathrm{CRT}_{\text {Del52 }}$ increases the fraction of monomers (Figures $4 \mathrm{~B}, 4 \mathrm{C}$ and $4 \mathrm{~F}$ ). Based on available crystal structures of CRT oligomers, we propose a model of $\mathrm{CRT}_{\text {Del52 }}$ dimer that is consistent with our experimental data, wherein dimer stabilizing interactions occur through $\mathrm{N}$-domains (C163-C163 disulfide and four ionic bridges involving D165) and through C-tails cross-linked by two C400-C404 disulfides (Figure $5 \mathrm{~A}$, and related discussion). The combinations of $\mathrm{D} 165 \mathrm{~K}$ with the triple cysteine mutant in 
$\mathrm{CRT}_{\text {Del52-3CA }}$ significantly increases the fraction of the monomeric form of the protein. This is not observed with $\mathrm{CRT}_{\text {De152-3CA/D166K }}$, which appears to induce structurally modified dimers (Figure 6B), which nonetheless are not compatible with $\mathrm{Mpl}$ binding and activation. Combined mutations that interfere with both dimerization interfaces $\left(\mathrm{CRT}_{\text {De152-3CA/D165K, }}\right.$ $\mathrm{CRT}_{\text {De152-3CA/D166K }}$ and $\left.\mathrm{CRT}_{\text {Del52-3CA/H170A }}\right)$ completely abrogate $\mathrm{CRT}_{\text {Del52-induced cell }}$ proliferation (Figures 7C and 7D).

Previous findings have indicated preferential binding of full-length CRT mutants to $\mathrm{Mpl}$ in transfected cells $(12,13,18-22)$. Here, we observed the binding of Mpl not only to the full-length $\mathrm{CRT}_{\text {Del52 }}$ (Figure $\mathrm{S} 3 \mathrm{~A}$ ) but also to its isolated C-terminal domain (Figures 2B and S4). This is direct evidence that the novel C-terminus of $\mathrm{CRT}_{\mathrm{Del} 52}$ confers the $\mathrm{Mpl}$ binding specificity. Cysteine residues within the mutant $\mathrm{C}$-domains are not absolutely required for $\mathrm{Mpl}$ binding either to the full-length $\mathrm{CRT}_{\text {Del52 }}$ or to its $\mathrm{C}$-domain (Figures $2 \mathrm{~B}$, 7E, and S4). However, mutations $\mathrm{C} 400 \mathrm{~A} / \mathrm{C} 404 \mathrm{~A}$ in $\mathrm{CRT}_{\text {Del52 }}$ reduced its ability to bind Mpl (Figure 7E-G). Additional disruption of intermolecular ionic interactions involving the globular N-domain that are predicted to stabilize $\mathrm{CRT}_{\text {De152 }}$ dimers (Figure 5A) further decreases $\mathrm{CRT}_{\text {Del52 }}$ binding to $\mathrm{Mpl}$ (Figures $7 \mathrm{~F}$ and $7 \mathrm{G}$ ) and eliminates its ability to induce proliferation of $\mathrm{Ba} / \mathrm{F} 3-\mathrm{Mpl}$ cells in a cytokine-independent manner (Figures 7C and 7D). These results indicate an important role of charged residues at dimerization interfaces in stabilizing functionally active conformations of $\mathrm{CRT}_{\mathrm{Del} 52 .}$

The formation of $\mathrm{Mpl}_{2}\left(\mathrm{CRT}_{\mathrm{Del} 52}\right)_{2}$ heterotetramers $(\sim 200 \mathrm{kDa})$ is previously suggested by size-exclusion chromatography (22). This could be achieved by binding of a $\left(\mathrm{CRT}_{\mathrm{Del} 52}\right)_{2}$ dimer (Figure 5A) to two Mpl molecules, inducing Mpl dimerization (Figure 8). In this type of model, two interaction interfaces are predicted to be key determinants of mutant CRT-Mpl binding: (i) C-domain-dependent interactions conferring the Mpl targeting specificity to CRT mutants (consistent with Figure 2) and (ii) glycan-dependent interactions between $\mathrm{N} 117$ of $\mathrm{Mpl}$ and carbohydrate-binding site of the mutant CRT (consistent with published data $(13,22)$ ). The loss of the majority or a subset of contacts with the first site are consistent with the impaired abilities of $\mathrm{CRT}_{\text {Del52 } 228}$ and $\mathrm{CRT}_{\mathrm{Del} 52 \triangle 36}$ to mediate cell proliferation (Figure 7A and (19)). Additionally, proliferation impairment observed with several dimer interface mutants (Figures 7C and 7D) is accompanied by altered disulfide/multimer patterns observed with those mutants (Figure 6). 
Overall, the findings of this study reveal that disulfide-mediated CRT multimerization is a fundamental feature of MPN CRT mutants. The mutant sequences not only directly induce multimer formation via C-terminal disulfides, but also appear to induce a conformational change in the globular N-domain that promotes multimerization through that domain. It is noteworthy that C-terminal truncations of CRT from some species induce its oligomerization propensity via interactions which most likely resemble those induced within the MPN mutants (38). Thus, cancers-linked mutations of CRT confer selective growth advantages to cells by inducing specificity for Mpl, by driving multimerization of the receptor, and by exploiting a natural dimerization interface of CRT, that becomes relevant under cell-stress conditions.

\section{STAR Methods}

\section{Healthy Donor and Patient Samples}

Blood was collected after written informed consent in accordance with the University of Michigan Institutional Review Board approved protocols for a myeloproliferative diseases repository (HUM0006778) or the University of Michigan Platelet Pharmacology and Physiology Platelet core (HUM00107120).

\section{DNA Constructs}

$\mathrm{CRT}_{\text {Ins5 }}$ (K385fs*47 (3)) was made using $\mathrm{CRT}_{\mathrm{WT}}$ (clone BC020493) as template by the QuickChange site-directed mutagenesis kit. $\mathrm{CRT}_{\mathrm{Del52}}$ (L367fs*46 (3)) was amplified from a patient cDNA. All primers, vectors and cloning methods used for CRT and Mpl cloning are specified in the SI Materials and Methods. Point mutants were generated using the QuickChange site-directed mutagenesis kit.

\section{Antibodies}

An anti-CRT mutant C-terminal antibody, anti-CRT $\left(\mathrm{C}_{\text {mut }}\right)$, was generated by GeneTel Laboratories using rabbits immunized with a 22-mer peptide (KMSPARPRTSCREACLQGWTEA) derived from the CRT mutant C-terminus and affinity purified. Other antibodies are specified in the SI Materials and Methods. 


\section{Platelet Isolation}

Whole blood from healthy donors and MPN patients was collected in ACD anticoagulant tubes (BD Biosciences) and platelets isolated as described previously (39) with some modifications described in the SI Materials and Methods.

\section{Immunoblotting and Immunoprecipitations}

Cell lines were lysed in lysis buffer (50 mM Tris $\mathrm{pH} 7.5,150 \mathrm{mM} \mathrm{NaCl}, 1 \%$ Triton X-100, $5 \mathrm{mM} \mathrm{CaCl}_{2}$ and protease inhibitor cocktail (Roche)) for $1 \mathrm{~h}$ at $4^{\circ} \mathrm{C}$. For native, reducing and non-reducing lysate blot experiments, transfected HEK293T cells were lysed in the presence of $10 \mathrm{mM}$ N-ethylmaleimide (Sigma). Protein concentration was measured using bicinchoninic acid assay (Pierce). For native blots, lysates were added to the loading buffer without reducing agents and SDS. For non-reducing blots, lysates were added to the loading buffer with SDS but without any reducing agents. For reducing blots, lysates were added to the loading buffer with SDS and reducing agent (65 mM DTT). Coimmunoprecipitation (IP) was performed using transfected HEK293T cells or $\mathrm{Ba} / \mathrm{F} 3 \mathrm{Mpl}$ CRT cells as described (40) with some modifications described in the SI Materials and Methods.

\section{Cytokine independent proliferation}

$\mathrm{Ba} / \mathrm{F} 3 \mathrm{Mpl}$ cells expressing CRT constructs were seeded at $1 \times 10^{6}$ cells $/ \mathrm{ml}$ in the absence of mouse IL-3. Further, the proliferation rate of different cell lines was calculated by counting the live cells using a hemocytometer.

Data Sharing Statement: Data will be deposited in Dryad following manuscript acceptance.

\section{Statistical analysis}

All statistical analysis was performed in GraphPad Prism (version 8.0c). 


\section{Acknowledgements}

We are grateful to all the blood donors for their contribution to this work. We thank the University of Michigan DNA Sequencing Core for DNA sequencing. We are grateful to Harihar Mohan, Angela Danielski and Maha Hamed for their contributions to the project. This work was funded by NIH grant (RO1 AI123957 to MR) and the University of Michigan Fast Forward Protein Folding Diseases Initiative. I.D.P. was supported by the NSF Division of Biological Infrastructure (award 1855425).

Authorship Contributions: AV helped design experiments, performed experiments, helped analyze data, and wrote sections of the manuscript. JG helped design experiments, performed experiments related to Figures 3 and 7, helped analyze data and edited the manuscript. MK collected patient samples, purified platelets and edited the manuscript. IP generated the structural model for the CRT-Mpl tetrameric complex, wrote some sections, and edited the manuscript. MT directs the University of Michigan MPN repository and edited the manuscript. SW helped construct and sequence some CRT variants. AB helped construct and sequence some CRT variants described in this manuscript. MR designed the study, analyzed data, and wrote sections of the manuscript.

Conflict of Interest Disclosures: The authors declare no conflicts of interest. 


\section{References}

1. Campbell PJ \& Green AR (2006) The myeloproliferative disorders. N Engl J Med 355(23):2452-2466.

2. Klampfl T, et al. (2013) Somatic mutations of calreticulin in myeloproliferative neoplasms. $N$ Engl J Med 369(25):2379-2390.

3. Nangalia J, et al. (2013) Somatic CALR mutations in myeloproliferative neoplasms with nonmutated JAK2. N Engl J Med 369(25):2391-2405.

4. Michalak M, Groenendyk J, Szabo E, Gold LI, \& Opas M (2009) Calreticulin, a multi-process calcium-buffering chaperone of the endoplasmic reticulum. Biochem J 417(3):651-666.

5. Raghavan M, Wijeyesakere SJ, Peters LR, \& Del Cid N (2013) Calreticulin in the immune system: ins and outs. Trends Immunol 34(1):13-21.

6. Chouquet A, et al. (2011) X-ray structure of the human calreticulin globular domain reveals a peptide-binding area and suggests a multi-molecular mechanism. PLoS One 6(3):e17886.

7. Kozlov G, et al. (2010) Structural basis of carbohydrate recognition by calreticulin. J Biol Chem 285(49):38612-38620.

8. Frickel EM, et al. (2002) TROSY-NMR reveals interaction between ERp57 and the tip of the calreticulin P-domain. Proc Natl Acad Sci U S A 99(4):1954-1959.

9. Nakamura K, et al. (2001) Functional specialization of calreticulin domains. $J$ Cell Biol 154(5):961-972.

10. Wijeyesakere SJ, Gafni AA, \& Raghavan M (2011) Calreticulin is a thermostable protein with distinct structural responses to different divalent cation environments. J Biol Chem 286(11):8771-8785.

11. Blees A, et al. (2017) Structure of the human MHC-I peptide-loading complex. Nature 551(7681):525-528.

12. Araki M, et al. (2016) Activation of the thrombopoietin receptor by mutant calreticulin in CALR-mutant myeloproliferative neoplasms. Blood 127(10):13071316.

13. Chachoua I, et al. (2016) Thrombopoietin receptor activation by myeloproliferative neoplasm associated calreticulin mutants. Blood 127(10):13251335.

14. Marty C, et al. (2016) Calreticulin mutants in mice induce an MPL-dependent thrombocytosis with frequent progression to myelofibrosis. Blood 127(10):13171324.

15. Shide K, et al. (2017) Calreticulin mutant mice develop essential thrombocythemia that is ameliorated by the JAK inhibitor ruxolitinib. Leukemia 31(5):1136-1144.

16. Balligand T, et al. (2019) Knock-in of murine Calr del52 induces essential thrombocythemia with slow-rising dominance in mice and reveals key role of Calr exon 9 in cardiac development. Leukemia.

17. Kollmann K, et al. (2017) A novel signalling screen demonstrates that CALR mutations activate essential MAPK signalling and facilitate megakaryocyte differentiation. Leukemia 31(4):934-944. 
18. Araki M, et al. (2018) Homomultimerization of mutant calreticulin is a prerequisite for MPL binding and activation. Leukemia.

19. Elf S, et al. (2016) Mutant Calreticulin Requires Both Its Mutant C-terminus and the Thrombopoietin Receptor for Oncogenic Transformation. Cancer Discov 6(4):368-381.

20. Elf S, et al. (2018) Defining the requirements for the pathogenic interaction between mutant calreticulin and MPL in MPN. Blood 131(7):782-786.

21. Masubuchi N, et al. (2019) Mutant calreticulin interacts with MPL in the secretion pathway for activation on the cell surface. Leukemia.

22. Pecquet C, et al. (2019) Calreticulin mutants as oncogenic rogue chaperones for TpoR and traffic-defective pathogenic TpoR mutants. Blood.

23. Baker SJ, Rane SG, \& Reddy EP (2007) Hematopoietic cytokine receptor signaling. Oncogene 26(47):6724-6737.

24. Moraga I, Spangler J, Mendoza JL, \& Garcia KC (2014) Multifarious determinants of cytokine receptor signaling specificity. Adv Immunol 121:1-39.

25. Syed RS, et al. (1998) Efficiency of signalling through cytokine receptors depends critically on receptor orientation. Nature 395(6701):511-516.

26. Mohan K, et al. (2019) Topological control of cytokine receptor signaling induces differential effects in hematopoiesis. Science 364(6442).

27. Matthews EE, et al. (2011) Thrombopoietin receptor activation: transmembrane helix dimerization, rotation, and allosteric modulation. FASEB J 25(7):2234-2244.

28. Charonis AS, Michalak M, Groenendyk J, \& Agellon LB (2017) Endoplasmic reticulum in health and disease: the 12th International Calreticulin Workshop, Delphi, Greece. J Cell Mol Med 21(12):3141-3149.

29. Arshad N \& Cresswell P (2018) Tumor-associated calreticulin variants functionally compromise the peptide loading complex and impair its recruitment of MHC-I. J Biol Chem 293(25):9555-9569.

30. Liu P, et al. (2020) Immunosuppression by Mutated Calreticulin Released from Malignant Cells. Mol Cell 77(4):748-760 e749.

31. Han L, et al. (2016) Calreticulin-mutant proteins induce megakaryocytic signaling to transform hematopoietic cells and undergo accelerated degradation and Golgimediated secretion. J Hematol Oncol 9(1):45.

32. Garbati MR, et al. (2016) Mutant calreticulin-expressing cells induce monocyte hyperreactivity through a paracrine mechanism. Am J Hematol 91(2):211-219.

33. Hammarstrom M, Woestenenk EA, Hellgren N, Hard T, \& Berglund H (2006) Effect of N-terminal solubility enhancing fusion proteins on yield of purified target protein. J Struct Funct Genomics 7(1):1-14.

34. DelProposto J, Majmudar CY, Smith JL, \& Brown WC (2009) Mocr: a novel fusion tag for enhancing solubility that is compatible with structural biology applications. Protein Expr Purif 63(1):40-49.

35. Moreau C, et al. (2016) Structures of parasite calreticulins provide insights into their flexibility and dual carbohydrate/peptide-binding properties. IUCrJ $3(\mathrm{Pt}$ 6):408-419.

36. Jeffery E, Peters LR, \& Raghavan M (2011) The polypeptide binding conformation of calreticulin facilitates its cell-surface expression under conditions of endoplasmic reticulum stress. J Biol Chem 286(4):2402-2415. 
37. Jorgensen CS, et al. (2003) Dimerization and oligomerization of the chaperone calreticulin. Eur J Biochem 270(20):4140-4148.

38. Rizvi SM, Mancino L, Thammavongsa V, Cantley RL, \& Raghavan M (2004) A polypeptide binding conformation of calreticulin is induced by heat shock, calcium depletion, or by deletion of the $\mathrm{C}$-terminal acidic region. $\mathrm{Mol} \mathrm{Cell}$ 15(6):913-923.

39. Greening DW, Sparrow RL, \& Simpson RJ (2011) Preparation of platelet concentrates. Methods Mol Biol 728:267-278.

40. Del Cid N, et al. (2010) Modes of calreticulin recruitment to the major histocompatibility complex class I assembly pathway. J Biol Chem 285(7):45204535.

41. Roy A, Kucukural A, \& Zhang Y (2010) I-TASSER: a unified platform for automated protein structure and function prediction. Nat Protoc 5(4):725-738. 


\section{Figures and legends}

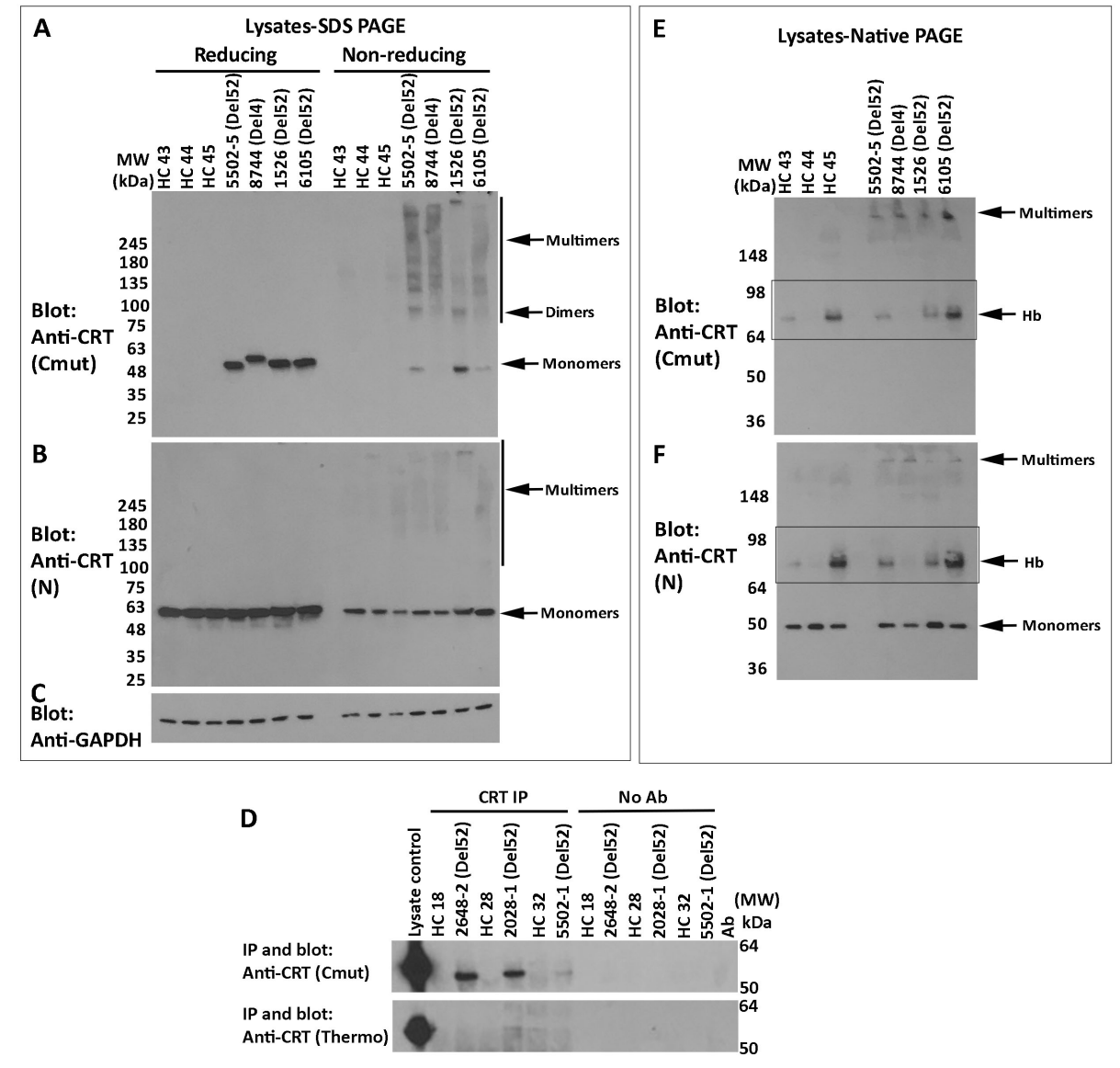

Fig. 1: Mutant CRTs form disulfide-stabilized multimers in MPN patient platelets and are detectable in MPN patient serum

A-C) Lysates from MPN patients or healthy donor platelets were probed by SDS-PAGE under reducing or non-reducing conditions (using 4-20\% gradient gels) followed by immunoblotting with anti-CRT $\left(\mathrm{C}_{\text {mut }}\right)(\mathrm{A})$, anti-CRT(N) antibodies (B) or anti-GAPDH as control (C). The same lysates were loaded in two blots (A and B) and B was re-probed with anti-GAPDH (C). D) CRT secretion in healthy donor or MPN patient serum was examined by immunoprecipitation/immunoblotting analyses with anti-CRT $\left(\mathrm{C}_{\mathrm{mut}}\right)$ (top panel) or antiCRT (Thermo Fisher) antibodies (bottom panel). No Ab lanes are controls in the absence of anti-CRT antibodies to assess non-specific precipitation. E and F) Representative immunoblots following native-PAGE ( $8 \%$ gels) of lysates from MPN patient platelets or healthy donor platelets, probed with the anti-CRT $\left(\mathrm{C}_{\text {mut }}\right)(\mathrm{E})$ and anti-CRT(N) antibodies (F). The band indicated as multimers are over-represented in CRT mutant platelet lysates. Boxes indicate hemoglobin $(\mathrm{Hb})$ contamination. In all panels, $\mathrm{HC}$ indicates healthy control samples; CRT mutant patient samples are indicated as Del52 or Del4 (characterized clinically as a 4 bp deletion in exon 9 resulting in a frameshift at K374, based on next generation sequencing. The predicted size of Del4 is 427 amino acids compared with 411 for De152). See also Figure $\mathrm{S} 1$ for more information on the specificity of anti-CRT $\left(\mathrm{C}_{\mathrm{mut}}\right)$ and Figure S2 for additional replicate blots of platelet lysates. 

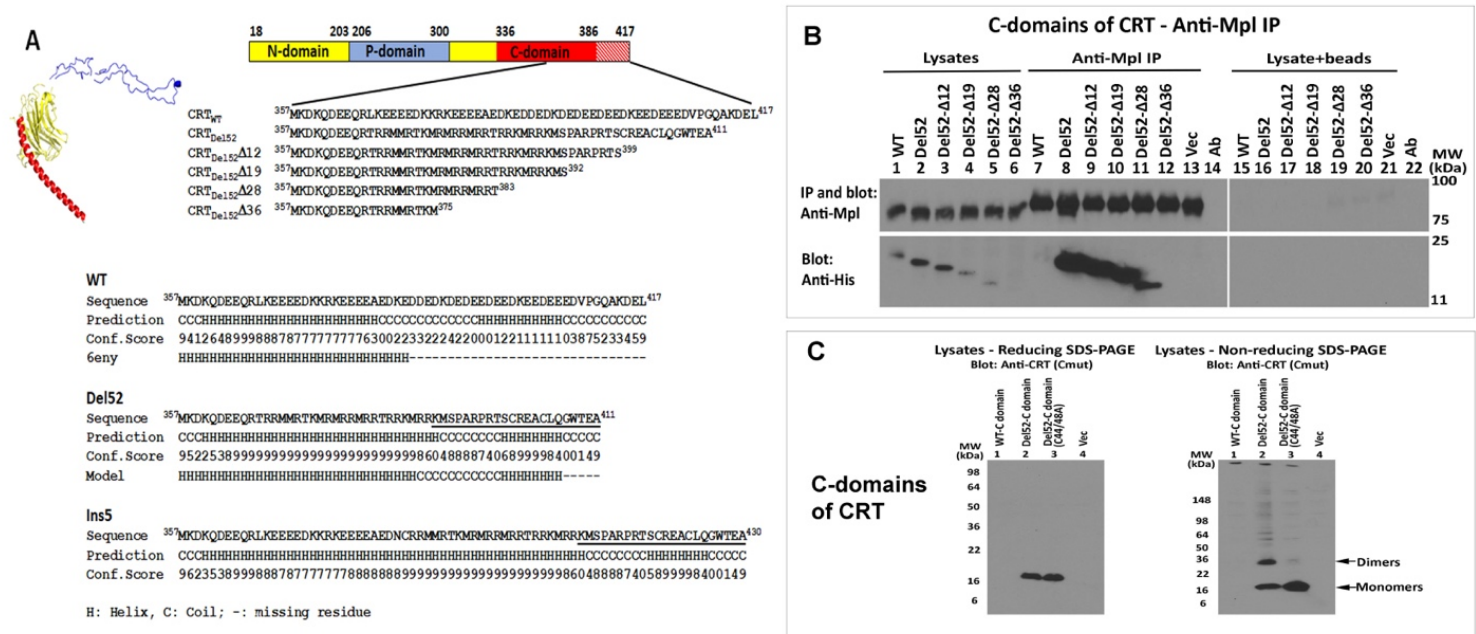

Fig. 2: The C-domain of $\mathrm{CRT}_{\mathrm{Del52}}$ confers specificity for $\mathrm{Mpl}$ and forms disulfidelinked dimers

A) Structure of $\mathrm{CRT}_{\mathrm{WT}}$ (PDB ID: 6eny, subunit G (11)) with a globular lectin-like domain (yellow, residues 18-203 from $\mathrm{N}$-domain and 301-335 from C-domain), a $\mathrm{P}$ domain (blue, residues 206-300), and an $\alpha$-helical (residues 336-386) C-domain (red). The C-terminus of the C-domain (red stripes, residues 387-417) is not resolved in the structure. Sequences of $\mathrm{CRT}_{\text {De152 }} \mathrm{C}$-domain truncation constructs used in this study are shown. For C-domain expression, MPN-mutated sequences are extended by 10 residues at the $\mathrm{N}$-terminus, to allow a common 10-mer sequence for the wild type and mutant $\mathrm{C}$-domain constructs. The lower panel shows indicated C-domain sequences (line 1), secondary structure predictions performed using I-TASSER (41) (lines 2-3), the secondary structure based on PDB: 6eny or the $\mathrm{CRT}_{\text {Del52 }}$ model discussed in this study (line 4). The sequence of the mutant-specific C-tail used to produce the anti-CRT $\left(\mathrm{C}_{\text {mut }}\right)$ antibody is underlined. B) HEK293T cells were transiently transfected with plasmids encoding N-terminal His-GB1-tagged C-domains of

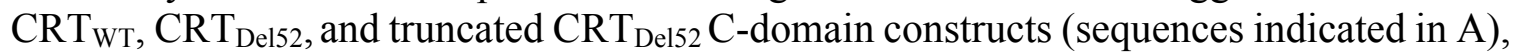
or a plasmid lacking CRT C-domain (Vec) along with N-terminal His-FLAG-tagged Mpl. Lysates from indicated cells were immunoprecipitated (indicated as IP) with anti-Mpl antibody or in the absence of anti-Mpl (marked as lysate+beads) and subsequent immunoblotting analyses were undertaken with the indicated antibodies. Data are representative of 3 independent experiments. C) HEK293T cells transiently transfected as indicated with plasmids encoding N-terminal His-GB1 tagged C-domains of CRT $\mathrm{WT}_{\mathrm{T}}$ $\left(\mathrm{CRT}_{\text {WT-C }}\right), \mathrm{CRT}_{\text {Del52 }}\left(\mathrm{CRT}_{\text {Del52-C }}\right)$, cysteines mutated $\mathrm{C}$-domain construct $\mathrm{CRT}_{\text {Del52- }}$ $\mathrm{C}(\mathrm{C} 44 \mathrm{~A} / \mathrm{C} 48 \mathrm{~A})$ or a plasmid lacking CRT C-domain (Vec). Cell lysates from indicated cells were separated by SDS-PAGE under reducing (12.5\% gels) (left panels) or non-reducing (4-20\% gradient gels) (right panels) conditions and immunoblotted with indicated antibodies. Data are representative of 4 independent experiments. See also Figure S3 for additional data on Mpl specificity for full-length mutant CRT and Figure S4 for data on the inability of $\mathrm{CRT}_{\mathrm{Del} 52} \mathrm{C}$-domain constructs to induce $\mathrm{Mpl}$ proliferation. 

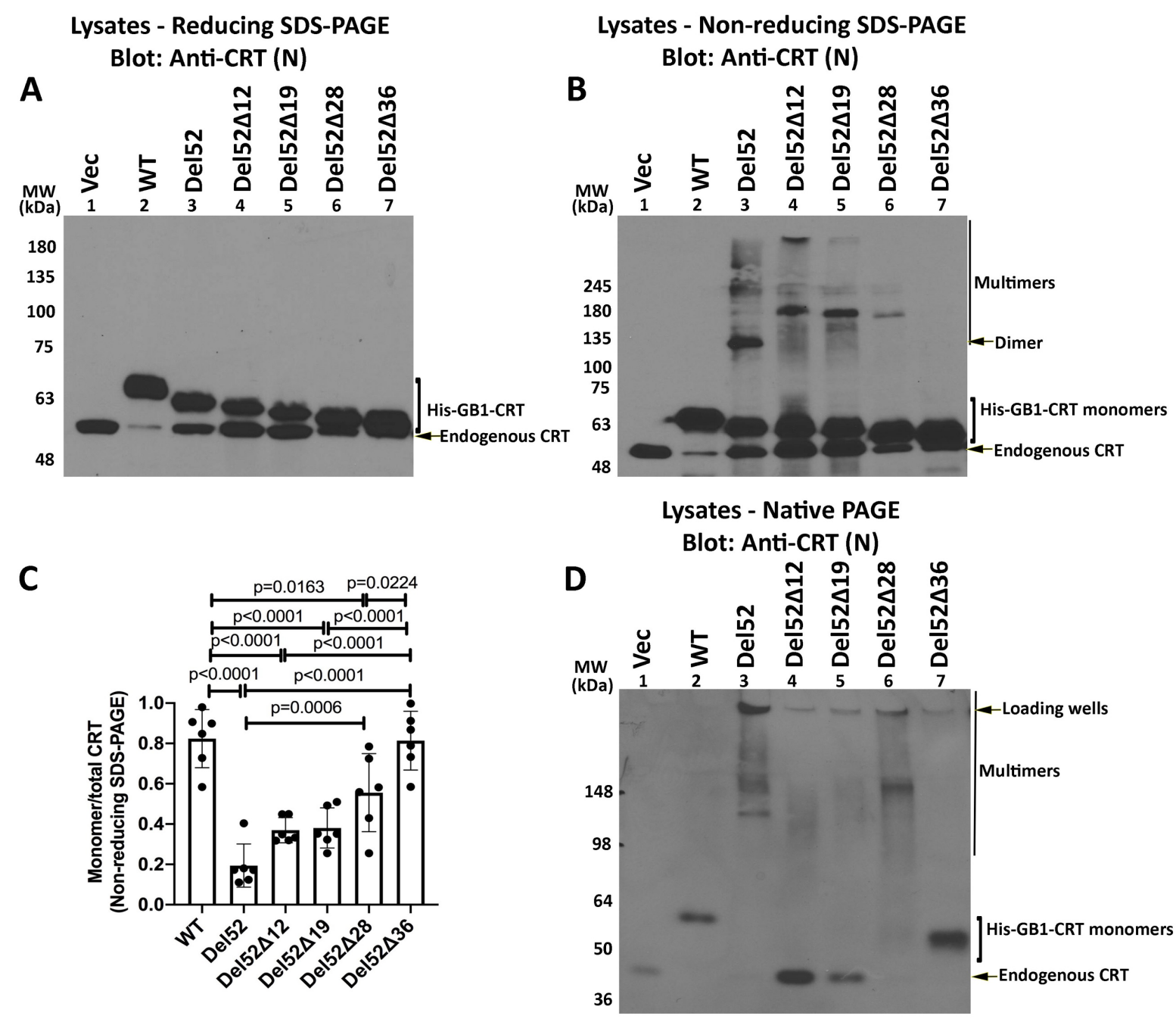

Fig. 3: Truncations of C-terminal cysteines of full-length $\mathrm{CRT}_{\text {Del52 }}$ alter but do not abrogate disulfide-linked interactions

HEK293T cells were transiently transfected with plasmids encoding N-terminal His-GB1 tagged full-length or C-terminally truncated $\mathrm{CRT}_{\text {Del52 }}$ constructs. Cell lysates from transfected cells were separated by SDS-PAGE under reducing (10\% gels) (A) or nonreducing (10\% gels) (B) conditions or by native-PAGE (4-20\% gradient gels) (D) and immunoblotted with the anti-CRT(N) antibody. Different amounts of lysates were loaded to achieve similar protein expression of different truncated constructs $\left(\mathrm{CRT}_{\mathrm{WT}}(0.5 \mu \mathrm{g}\right.$

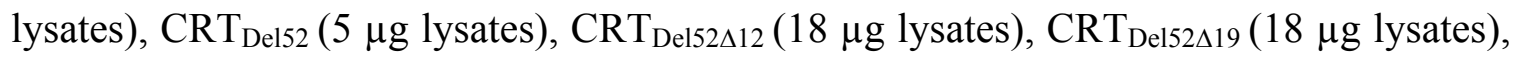
$\mathrm{CRT}_{\text {Del52 } 228}\left(3 \mu \mathrm{g}\right.$ lysates), $\mathrm{CRT}_{\text {Del52 } 236}(1.8 \mu \mathrm{g}$ lysates), or a plasmid lacking CRT (Vec) (10 $\mu$ g lysates). The endogenous CRT band serves as the lysate loading controls. Species consistent with the size of endogenous CRT, His-GB1-CRT monomers, dimers, multimers and loading wells are indicated. Quantification of CRT monomer/monomer+multimer (total) bands from B is shown in C, averaged over 6 independent blots from 5 independent transfections. Data show mean $\pm \mathrm{SD}$, with statistical significance assessed via ordinaryone-way ANOVA. 


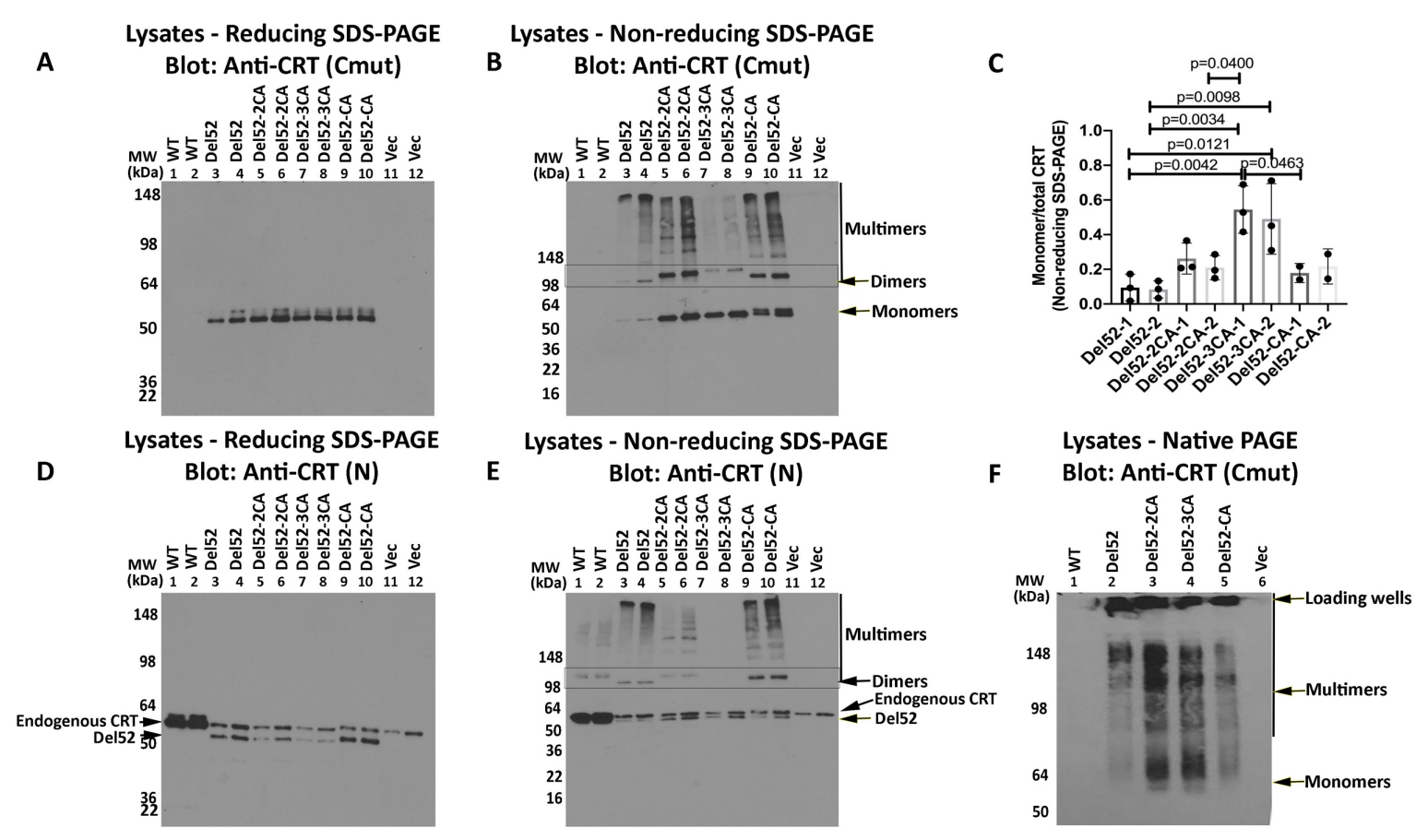

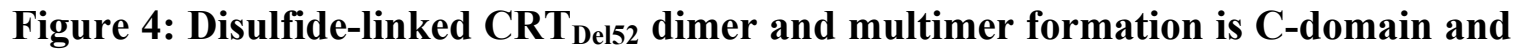
N-domain dependent

HEK293T cells were transiently transfected with plasmids encoding untagged full-length CRT $_{\text {WT }}, \quad$ CRT $_{\text {Del52 }}, \quad$ CRT $_{\text {Del52-2CA }} \quad\left(\right.$ CRT $\left._{\text {Del52(C400A/C404A })}\right), \quad$ CRT $_{\text {Del52-3CA }}$ $\left(\mathrm{CRT}_{\text {Del52(C163A/C400A/C404A) }}\right)$, $\mathrm{CRT}_{\text {Del52-CA }}\left(\mathrm{CRT}_{\text {Del52(C163A) }}\right)$ or plasmid lacking CRT (Vec). Cell lysates from indicated cells with two lysate amounts (A-B, D-E) were separated by SDS-PAGE under reducing (8\% gels) (A, D) or non-reducing (4-20\% gradient gels) (B, E) conditions and immunoblotted with indicated antibodies. Full gel panels are shown. Data are representative of three independent experiments for $\mathrm{CRT}_{\mathrm{WT}}, \mathrm{CRT}_{\text {De152, }} \mathrm{CRT}_{\text {De152-2CA, }}$,

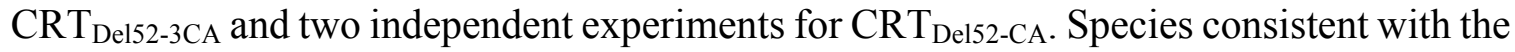
size of CRT monomers, dimers and multimers are indicated. In panel D where CRT $\mathrm{WT}_{\mathrm{W}}$ and $\mathrm{CRT}_{\text {Del52 }}$ constructs are resolved, the migration position of each are indicated as

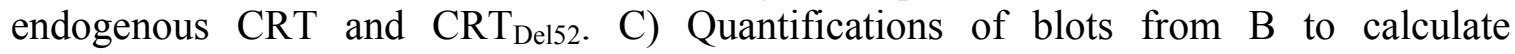
monomer/monomer+multimer (total) mutant CRT following non-reducing SDS-PAGE are shown in $\mathrm{C}$, averaged over 3 independent experiments (two protein loads each). Consistent ratios are quantified at two different protein loads of each construct (labeled as 1 or 2). Data show mean $\pm \mathrm{SD}$, with statistical significance assessed via ordinary-one-way ANOVA. F) Cell lysates from indicated transfected cells were separated by native-PAGE (8\% gels) and immunoblotted with indicated antibody. Bands corresponding to CRT $_{\text {Del52 }}$ monomers, multimers and loading wells are indicated. See also Figure S5 for disulfidelinked multimer formation by $\mathrm{CRT}_{\text {Ins5. }}$. 
A
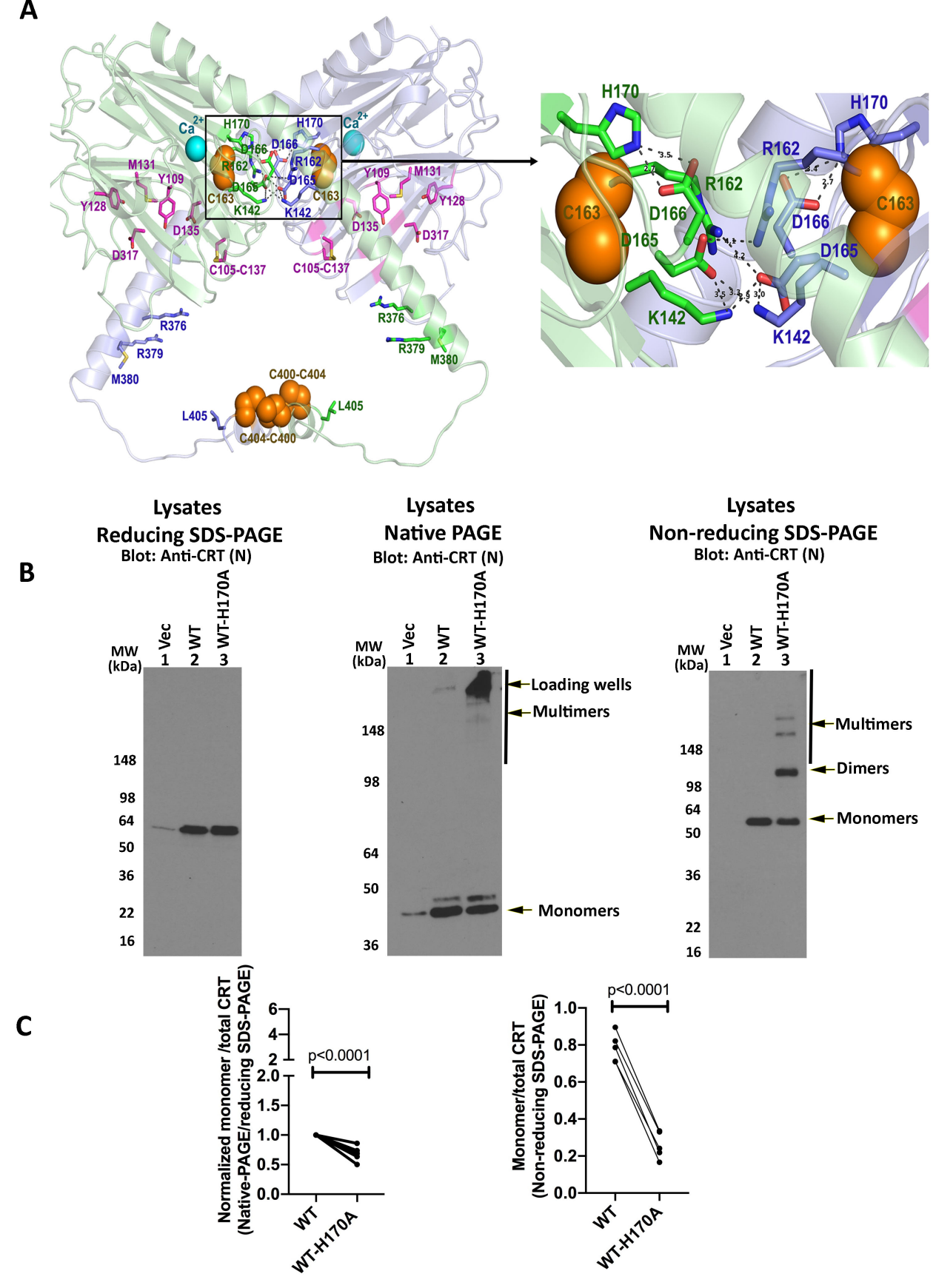

Fig. 5: A working model for a $\mathrm{CRT}_{\text {Del52 }}$ dimer and assessment of H170-regulated wild type CRT multimerization

A) A molecular model of $\mathrm{CRT}_{\mathrm{Del} 52}$ dimer is primarily based on the crystal structure of the 10-mer complex of CRT D71K mutant (PDB ID: 5lk5, subunits E and G (35)). Subunit 1 is colored light green and subunit 2 light blue. Each subunit contains a globular N-domain and a C-domain comprising long proximal and short distal $\alpha$-helices connected by a 14 residue loop. The $\mathrm{P}$-domain is omitted. $\mathrm{Ca}^{2+}$-ions bound to the high-affinity site are shown by cyan spheres. Residues from the carbohydrate recognition site (7) are shown by purple sticks (for $\mathrm{C} \alpha$-atoms). Cysteines participating in the formation of predicted intermolecular 
disulfide bonds (C163-C163 and two C400-C404) are shown by orange spheres. To form a $\mathrm{C} 163-\mathrm{C} 163$ disulfide bond, loops have to be rearranged to decrease the distance between cysteines. Dimers are also stabilized by intermolecular hydrogen bonds (shown by gray dashes, distances $<3.0 \AA$ ) and ionic interactions (shown by gray dashes, distances $<4.5$ $\AA$ ) that are formed between charged residues from the N-domain dimerization interface: D165, K142, and R162 (residues are shown by sticks colored green for subunit E and blue for subunit $\mathrm{G}$ ). Inset highlights interactions at the contact interface. Molecular graphic representations were generated by PyMOL. See also Figure S6 for models of the structural organization of CRT dimers into higher order multimers. B) HEK293T cells were transiently transfected with plasmids encoding untagged full-length $\mathrm{CRT}_{\mathrm{WT}}, \mathrm{CRT}_{\mathrm{WT}-\mathrm{H} 170 \mathrm{~A}}$ or plasmid lacking CRT (Vec). Cell lysates from indicated cells were separated under reducing SDS-PAGE ( $8 \%$ gel, left panel) or native-PAGE ( $8 \%$ gel, middle panel) or nonreducing SDS-PAGE (4-20\% gradient gel, right panel) conditions and immunoblotted with anti-CRT (N) antibody. C) Left panel: Monomer bands for each construct from the nativePAGE immunoblots were quantified and normalized relative to the total CRT signal from parallel SDS-PAGE immunoblots, averaged over 6 independent blots from 4 independent transfections. Data show mean $\pm \mathrm{SD}$, the normalized signals were log transformed and the statistical significance was assessed via one sample t-test. Right panel: Quantifications of monomer/monomer+multimer (total) mutant CRT bands from non-reducing SDS-PAGE immunoblots, averaged over 5 independent blots from 4 independent transfections. Data show mean \pm SD and the statistical significance was assessed via a paired t-test. 


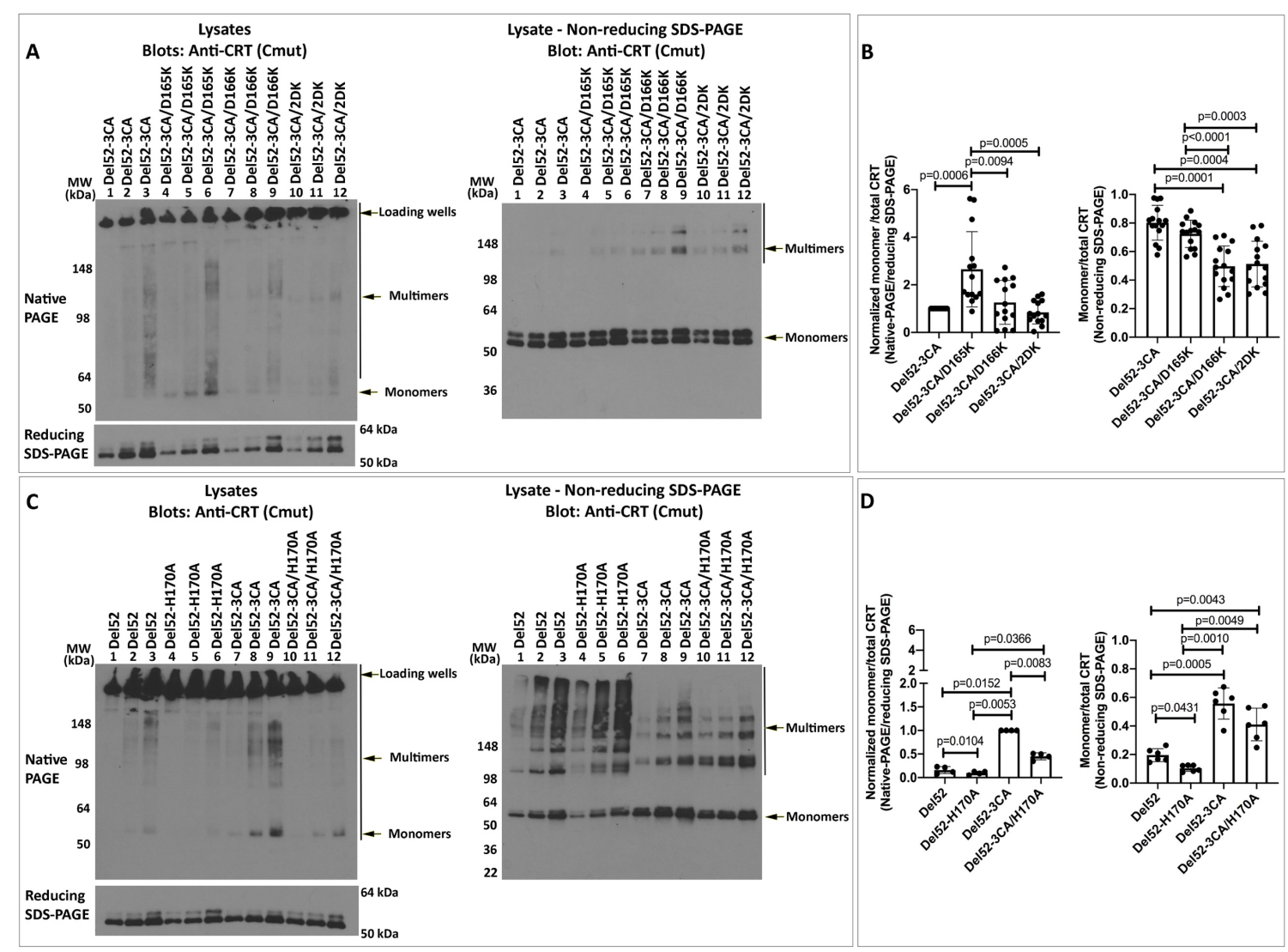

Fig. 6: N-domain dimer interface residues influence CRT multimerization

HEK293T cells were transiently transfected as indicated with plasmids encoding untagged full-length $\quad \mathrm{CRT}_{\text {Del52-3CA }} \quad\left(\mathrm{CRT}_{\text {Del52(C163A/C400A/C404A })}\right), \quad \mathrm{CRT}_{\text {Del52-3CA/D165K }}$ $\left(\mathrm{CRT}_{\text {Del52(C163A/C400A/C404A/D165K) }}\right), \mathrm{CRT}_{\text {Del52-3CA/D166K }}\left(\mathrm{CRT}_{\text {Del52(C163A/C400A/C404A/D166K) }}\right)$, and $\mathrm{CRT}_{\text {Del52-3CA/2DK }} \quad\left(\mathrm{CRT}_{\text {Del52(C163A/C400A/C404A/D165K/D166K) }}\right), \mathrm{CRT}_{\text {Del52-H170A }}$ or CRT $_{\text {Del52- }}$

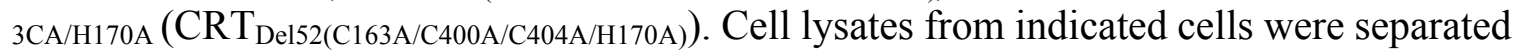
by native-PAGE ( $8 \%$ gels) (A and $\mathrm{C}$, top left panels) or SDS-PAGE under reducing ( $8 \%$ gels) (A and C, bottom left panel) or non-reducing (4-20\% gradient gels) (A and C, right panels) and immunoblotted with the anti-CRT $\left(\mathrm{C}_{\mathrm{mut}}\right)$ antibody. $\mathrm{B}$ and $\left.\mathrm{D}\right)$ Left panels: Mutant CRT monomer bands from native-PAGE immunoblots in panels $\mathrm{A}$ and $\mathrm{C}$ were normalized relative to the corresponding total CRT signal from the reducing SDS-PAGE immunoblots. Data show mean $\pm \mathrm{SD}$, the normalized signals were log transformed and the statistical significance was assessed via RM one-way ANOVA analysis. B and D) Right Panels: Quantification of CRT monomer/ monomer+multimer (total) bands from the nonreducing SDS-PAGE immunoblots. Data show mean $\pm \mathrm{SD}$ and the statistical significance was assessed via RM one-way ANOVA analysis. Data were averaged over 5 independent blots from 5 independent transfections (B) or 2 independent blots from 2 independent transfections (D), each with 2-3 protein loads. See also Figure S7 for multimer formation by $\mathrm{CRT}_{\mathrm{Del} 2 \mathrm{2}-\mathrm{D} 165 \mathrm{~K}}$ and $\mathrm{CRT}_{\mathrm{Del} 52-\mathrm{D} 166 \mathrm{~K}}$. 

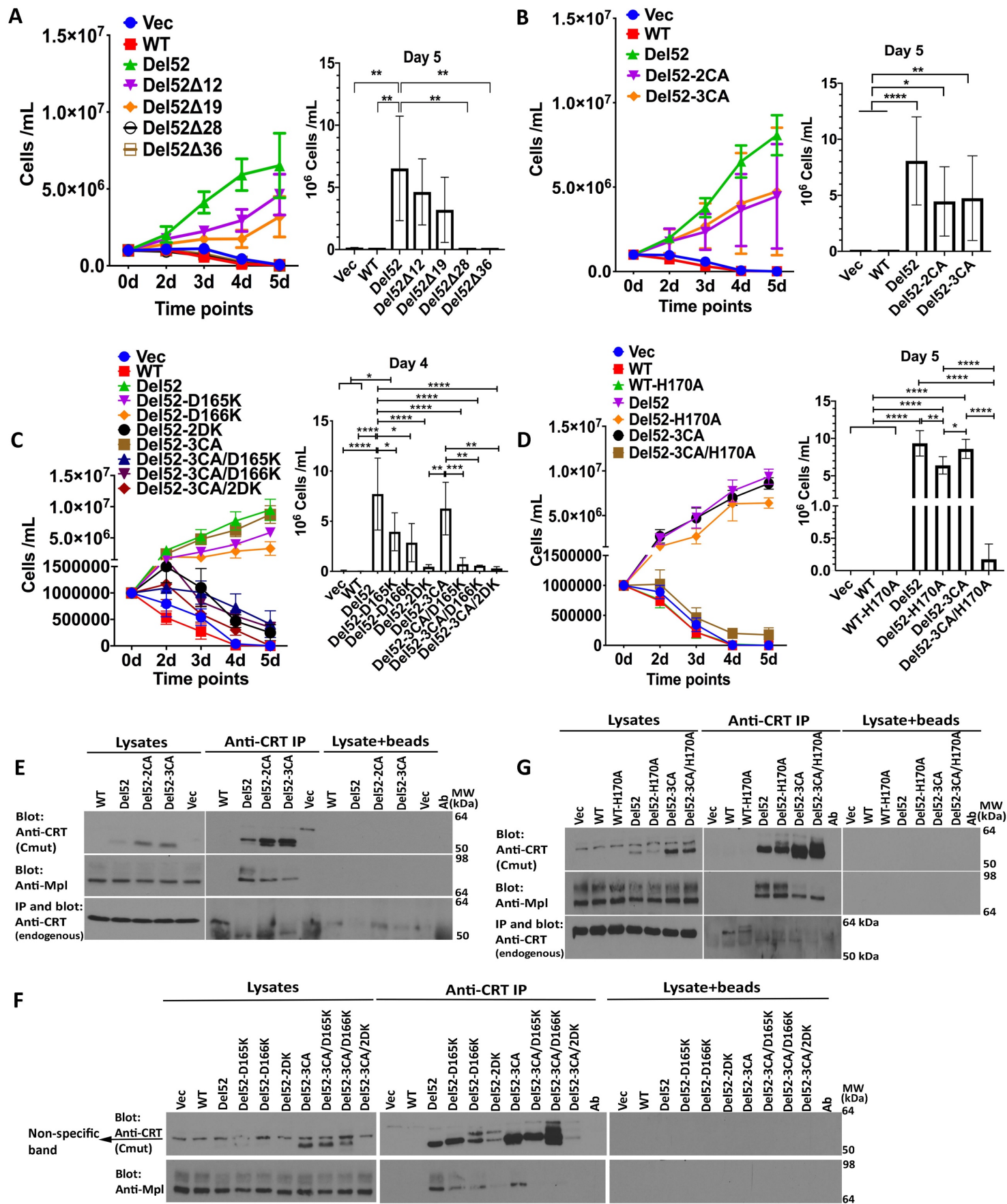

Fig. 7: Large C-domain truncations or combined N-domain and C-domain dimer interface mutations are required to abrogate $\mathrm{CRT}_{\text {Del52-mediated cell proliferation }}$

$\mathrm{Ba} / \mathrm{F} 3-\mathrm{Mpl}$ cells as specified were transduced with retroviruses $(\mathrm{Ba} / \mathrm{F} 3-\mathrm{Mpl})$ encoding full-

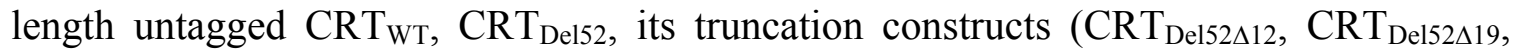
$\left.\mathrm{CRT}_{\text {Del52 } 228}, \mathrm{CRT}_{\text {Del52 } \triangle 36}\right)$, point mutants $\left(\mathrm{CRT}_{\text {Del52-2CA }}\left(\mathrm{CRT}_{\text {Del52(C400A/C404A)), }} \mathrm{CRT}_{\text {Del52-3CA }}\right.\right.$ (CRT Del52(C163A/C400A/C404A) $), \quad$ CRT $_{\text {Del52-D165K, }} \quad$ CRT $_{\text {Del52-D166K },} \quad$ CRT $_{\text {Del52-2DK }}$ $\left(\mathrm{CRT}_{\text {Del52(D165/166K) })}\right), \quad \mathrm{CRT}_{\text {Del52-3CA/D165K }} \quad\left(\mathrm{CRT}_{\text {Del52(C163A/C400A/C404A/D165K) }}\right), \quad \mathrm{CRT}_{\text {Del52- }}$ 3CA/D166K

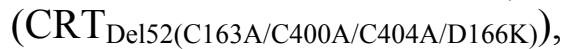

$\mathrm{CRT}_{\text {Del52-3CA/2DK }}$ $\left(\mathrm{CRT}_{\text {Del52(C163A/C400A/C404A/D165K/D166K) }),} \mathrm{CRT}_{\text {WT-H170A }}, \mathrm{CRT}_{\text {Del52-H170A, }}, \mathrm{CRT}_{\text {Del52-3CA/H170A }}\right.$ 
$\left.\left(\mathrm{CRT}_{\mathrm{Del52}(\mathrm{C} 163 \mathrm{~A} / \mathrm{C} 400 \mathrm{~A} / \mathrm{C} 404 \mathrm{~A} / \mathrm{H} 170 \mathrm{~A})}\right)\right)$, or control viruses and used for the subsequent analyses. A-D) Cytokine-independent proliferation of $\mathrm{Ba} / \mathrm{F} 3-\mathrm{Mpl}$ cells. Cell proliferation was measured as described in Figure S4 legend. Data are averaged from three separate viral transductions of $\mathrm{Ba} / \mathrm{F} 3-\mathrm{Mpl}$ cells and a total of 5 independent proliferation experiments (A), 8 separate viral transductions of $\mathrm{Ba} / \mathrm{F} 3-\mathrm{Mpl}$ cells, and a total of 10-13 independent experiments (B), 2-3 separate viral transductions of $\mathrm{Ba} / \mathrm{F} 3-\mathrm{Mpl}$ cells, and a total of 3-6 experiments $(\mathrm{C})$ or two separate retroviral transductions of $\mathrm{Ba} / \mathrm{F} 3-\mathrm{Mpl}$ cells, and a total of 5 independent experiments (D). Mean \pm SD is shown, with statistical significance assessed via ordinary-one-way ANOVA from the indicated days of proliferation assay. Statistically significant means are indicated as $* p<0.05, * * p<0.01, * * * p<0.001$, and $* * * * p<0.0001$. E-G) Lysates from $\mathrm{Ba} / \mathrm{F} 3-\mathrm{Mpl}$ cells expressing indicated constructs or control cells expressing $\mathrm{Mpl}$ alone (Vec) were directly loaded for immunoblotting analyses (labeled as lysates) or immunoprecipitated (indicated as IP) with anti-CRT $\left(\mathrm{C}_{\text {mut }}\right.$ ) antibody (for $\mathrm{CRT}_{\text {Del52 }}$ and its variants) or with anti-CRT(Thermo) antibody (for $\mathrm{CRT}_{\mathrm{WT}}$ ) and subsequent immunoblotting was undertaken with the indicated antibodies. Results are representative of 3 (E and F) or $4(\mathrm{G})$ independent experiments. Non-specific interactions in the absence of primary antibody are shown by the lysate+ beads lanes. See also Figure S8 for proliferation induction by His-GB1-tagged versions of the $\mathrm{CRT}_{\text {Del52 }}$ truncation mutants. 


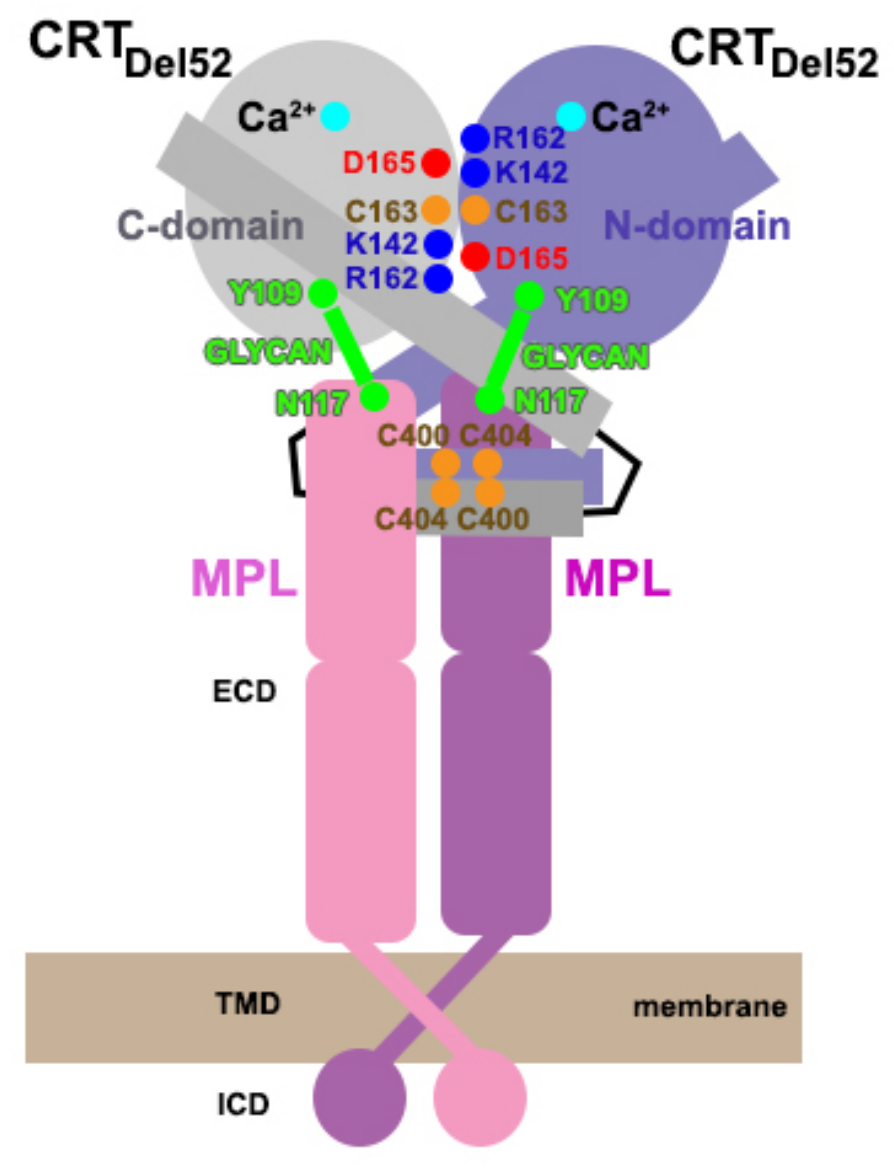

Figure 8: Schematic representation of a proposed $\mathrm{Mpl}_{2}\left(\mathrm{CRT}_{\text {Del52 }}\right)_{2}$ complex. Mpl activation is mediated by covalently-linked $\mathrm{CRT}_{\text {Del } 52}$ dimers stabilized by disulfides and ionic interactions at $\mathrm{C}$-domain and $\mathrm{N}$-domain dimerization interfaces. The $\mathrm{C}$-domain-of $\mathrm{CRT}_{\text {Del52 }}$ also contributes to the specificity of Mpl binding (Figure 2), in addition to generic glycan-binding site residues of CRT that are previously shown to mediate Mpl recruitment $(13,20,21)$. 